QUARTERLY OF APPLIED MATHEMATICS

VOLUME LXXI, NUMBER 1

MARCH 2013, PAGES 37-67

S $0033-569 X(2012) 01271-6$

Article electronically published on August 27, 2012

\title{
ON THE INCOMPRESSIBLE LIMIT PROBLEMS FOR MULTICOMPONENT REACTIVE FLOWS
}

\author{
BY \\ YOUNG-SAM KWON (Department of Mathematics, Dong-A University, Busan 604-714, Korea) \\ AND \\ KONSTANTINA TRIVISA (Department of Mathematics, University of Maryland, College Park, \\ Maryland 20742)
}

\begin{abstract}
Multicomponent reactive flows are associated with a variety of phenomena and processes: pollutant formation, biotechnology, fuel droplets in combustion, sprays, astrophysical plasma. Analyzing the physical regimes associated with various processes unfolds complex chemistry mechanisms and detailed transport phenomena. Many interesting problems in that context involve the behavior of solutions to the governing equations for multicomponent reactive flows as certain parameters vanish or become infinity. This work establishes rigorously the incompressible limit for weak solutions to multicomponent reactive flows. The analysis treats the cases of both bounded and unbounded domains.
\end{abstract}

1. Introduction. Multicomponent reactive flows arise in the sciences and engineering and are associated with a variety of phenomena and processes: pollutant formation, biotechnology, fuel droplets in combustion, sprays, and astrophysical plasma. Analyzing the physical regimes associated with various processes unfolds complex chemistry mechanisms and detailed transport phenomena. Many interesting problems in that context involve the behavior of solutions to the governing equations for multicomponent reactive flows as certain parameters vanish or become infinity. The objective of this work is to investigate singular limits for such complex flows. The analysis treats the cases of both bounded and unbounded domains.

Received March 1, 2011.

2000 Mathematics Subject Classification. 35B40, 35D05, 76N10, $35 \mathrm{~B} 45$.

Key words and phrases. Incompressible limits, low Mach number, reactive flows, compressible and viscous fluid, reversible reaction.

The work of Y.-S. Kwon was supported in part by the Basic Science Research Program through the National Research Foundation of Korea (NRF) funded by the Ministry of Education, Science and Technology (20110005336).

The work of K. Trivisa was supported in part by the National Science Foundation under the Grants DMS-1109397, DMS-0807815, DMS-0405853 and PECASE DMS-0239063.

E-mail address: ykwon@dau.ac.kr

E-mail address: trivisa@math.umd.edu

(C) 2012 Brown University Reverts to public domain 28 years from publication 
The state of multicomponent reactive flows is, in general, characterized by the total mass density $\varrho=\varrho(t, x)$, the velocity field $\mathbf{u}=\mathbf{u}(t, x)$, the absolute temperature $\vartheta=$ $\vartheta(t, x)$, and the species mass fractions $Y_{k}=Y_{k}(t, x), k=1, \ldots, N$, depending on the time $t \in(0, T)$ and the Eulerian spatial coordinate $x \in \Omega \subset \mathbb{R}^{3}$.

The governing equations for multicomponent flows are expressed as the conservation of mass, momentum, energy, and conservation of species mass (cf. Feireisl, Petzeltová and Trivisa 9, Giovangigli [11, Chapter 2, Section 2.2]):

$$
\begin{gathered}
\partial_{t} \varrho+\operatorname{div}_{x}(\varrho \mathbf{u})=0, \\
\partial_{t}(\varrho \mathbf{u})+\operatorname{div}_{x}(\varrho \mathbf{u} \otimes \mathbf{u})+\frac{1}{\mathrm{Ma}^{2}} \nabla_{x} p=\operatorname{div}_{x} \mathbb{S}, \\
\partial_{t}(\varrho s)+\operatorname{div}_{x}(\varrho s \mathbf{u})+\operatorname{div}_{x}\left(\frac{\mathbf{q}}{\vartheta}-\sum_{k=1}^{N} s_{k} \mathcal{F}_{k}\right)=\sigma, \\
\partial_{t}\left(\varrho Y_{k}\right)+\operatorname{div}_{x}\left(\varrho Y_{k} \mathbf{u}\right)=\operatorname{div}_{x}\left(\mathcal{F}_{k}\right)+\varrho \omega_{k}, k=1, \ldots, N, \\
\partial_{t} \int_{\Omega}\left(\frac{\mathrm{Ma}^{2}}{2} \varrho|\mathbf{u}|^{2}+\varrho e\right) \mathrm{d} x=0,
\end{gathered}
$$

where $p$ is the pressure, $\mathbb{S}$ stands for the viscous stress tensor, $\mathbf{q}$ is the heat flux, $\mathcal{F}_{k}$ denotes the diffusion flux and $\omega_{k}$ is the production rate of the $k$-th species with $\omega_{k}=$ Da $\bar{\omega}_{k}$, whereas $h_{k}, k=1, \ldots, N$ denote the species formation enthalpies, which in the present context are assumed to be constants.

Motivated by several recent studies devoted to the scale analysis as well as numerical experiments related to the proposed model (see Klein et al. [14]), our aim is to provide rigorous analysis, taking into account the principles of continuum physics.

1.1. Transport Fluxes. The transport fluxes $\mathbb{S}, \mathbf{q}, \mathcal{F}$ are given as follows.

[F1] The viscous stress tensor $\mathbb{S}$ is determined through Newton's rheological law

$$
\mathbb{S}=\mu\left(\nabla_{x} \mathbf{u}+\nabla_{x}^{\perp} \mathbf{u}-\frac{2}{3} \operatorname{div}_{x} \mathbf{u I}\right)+\eta \operatorname{div}_{x} \mathbf{u} \mathbb{I},
$$

where $\mu>0, \eta \geq 0$ are respectively the shear and bulk viscosity coefficients.

[F2] The heat flux $\mathbf{q}$ is given by Fourier's law

$$
\mathbf{q}=-\kappa \nabla_{x} \vartheta
$$

with the heat conductivity coefficient $\kappa>0$.

[F3] The species diffusion fluxes are determined through Fick's empirical law

$$
\mathcal{F}_{k}=D_{k} \nabla_{x} Y_{k}, k=1, \ldots, N,
$$

where $D_{k}$ stands for the diffusion coefficient of the $k$-th species.

The model is consistent with the principle of mass conservation, which means

$$
\sum_{k=1}^{N} Y_{k}=1, \sum_{k=1}^{N} \omega_{k}=0,
$$

and the second law of thermodynamics requiring the entropy production to be nonnegative. 
Here, and in what follows, we denote the dimensionless parameters Ma and Da by the Mach, Damköhler numbers, respectively (see Klein et al. [14]). We now take

$$
\mathrm{Ma}=\epsilon, \mathrm{Da}=\epsilon^{-1} .
$$

With this scaling, the system (1.1)-(1.5) reads

$$
\left\{\begin{array}{c}
\partial_{t} \varrho+\operatorname{div}_{x}(\varrho \mathbf{u})=0 \\
\partial_{t}(\varrho \mathbf{u})+\operatorname{div}_{x}(\varrho \mathbf{u} \otimes \mathbf{u})+\frac{1}{\epsilon^{2}} \nabla_{x} p=\operatorname{div}_{x} \mathbb{S} \\
\partial_{t}(\varrho s)+\operatorname{div}_{x}(\varrho s \mathbf{u})+\operatorname{div}_{x}\left(\frac{\mathbf{q}}{\vartheta}-\sum_{k=1}^{N} s_{k} \mathcal{F}_{k}\right)=\sigma \\
\partial_{t}\left(\varrho Y_{k}\right)+\operatorname{div}_{x}\left(\varrho Y_{k} \mathbf{u}\right)=\operatorname{div}_{x}\left(\mathcal{F}_{k}\right)+\epsilon^{-1} \varrho \bar{\omega}_{k}, k=1, \ldots, N, \\
\partial_{t} \int_{\Omega}\left(\frac{\epsilon^{2}}{2} \varrho|\mathbf{u}|^{2}+\varrho e\right) \mathrm{d} x=0
\end{array}\right.
$$

where $\omega_{k}$ is defined by

$$
\omega_{k}=\frac{1}{\epsilon} \bar{\omega}_{k}
$$

We will investigate the limit

$$
\varrho_{\epsilon} \rightarrow \bar{\varrho}, \mathbf{u}_{\epsilon} \rightarrow \mathbf{U}, \frac{\vartheta_{\epsilon}-\bar{\vartheta}}{\epsilon} \rightarrow \Theta, \frac{Y_{k}^{\epsilon}}{\epsilon} \rightarrow Y_{k}
$$

as $\epsilon$ tends to 0 in the suitable sense. Our goal is to show that $\left\{\mathbf{U}, \Theta, Y_{k}\right\}$ represents a solution of the following system:

$$
\left\{\begin{array}{c}
\operatorname{div}_{x} \mathbf{U}=0, \\
\bar{\varrho}\left(\partial_{t} \mathbf{U}+\mathbf{U} \cdot \nabla_{x} \mathbf{u}\right)+\nabla_{x} P=\bar{\mu} \Delta \mathbf{U}, \\
\bar{\varrho} \bar{c}_{p}\left(\partial_{t} \Theta+\mathbf{U} \cdot \nabla_{x} \Theta\right)=\operatorname{div}_{x}\left(\bar{\kappa} \nabla_{x} \Theta\right), \\
\bar{\varrho}\left(\partial_{t} Y_{k}+\mathbf{U} \cdot \nabla_{x} Y_{k}\right)=\operatorname{div}_{x}\left(D_{k}(\bar{\vartheta}) \nabla_{x} Y_{k}\right),
\end{array}\right.
$$

for each $k=1,2, \ldots, N$.

We first notice that the global-in-time existence solutions for system (1.1)-(1.4), supplemented with physically relevant constitutive relations, has been studied by Feireisl, Petzeltova, and Trivisa [9. Recently low Mach number problems have been achieved by many authors, as a first result, Klainerman, Majda [13] for the Euler equations and then Lions, Masmoudi [15] extended their work based on another approach for compressible Navier-Stokes equations. Similar results with the same strategy of Lions, Masmoudi [15] include the recent progress by Novotný, Feiriesl [6, 7, for the full Navier-Stokes Fourier system and by Feireisl and Petzeltova 8 for the reactive flows. In this paper, we investigate the low Mach number limit problem for multicomponent reactive flows governed 
by the system (1.1) - (1.4) on bounded domains with Dirichlet boundary condition and on unbounded domains.

The outline of this article is as follows: In Section 2 we present the fundamental principles of thermodynamics and the constitutive relations satisfied by various nonlinear quantities in the system. In Section 3 we introduce the notion of weak solutions for the NSF system and limiting system (1.14). In Section 4 we present the main results of the article on the low Mach number problems on bounded domains with Dirichlet boundary condition and unbounded domains. In Section [5 we present the proof of the low Mach number problem for the multicomponent reactive flows. In Section 6 we give the rigorous proof of the low Mach number problem for the multicomponent reactive flows on unbounded domains in the spirit of Feireisl [4].

2. Constitutive relations. The constitutive equations can be expressed as typically non-linear functions relating the pressure $p$, internal energy $e$ and entropy $s$ in (1.1)-(1.4) to the macroscopic variables $\varrho, \mathbf{u}, \vartheta$, and $Y_{k}, k=1, \ldots, N$.

(a) The total energy $E$ can be written in the form

$$
E=\frac{1}{2}|\mathbf{u}|^{2}+e
$$

(b) In accordance with the basic principles of statistical mechanics (cf. Gallavotti [10]), the specific internal energy $e$ is related to the pressure $p$ and the species mass fractions $Y_{k}, k=1, \ldots, n$, through Gibb's equation

$$
\vartheta \mathrm{D} s=\mathrm{D} e+p \mathrm{D}\left(\frac{1}{\varrho}\right)-\sum_{k=1}^{N} g_{k} \mathrm{D} Y_{k},
$$

where $\mathrm{D}$ denotes the differential with respect to the state variables $\varrho, \vartheta, Y_{k}$, and

$$
g_{k}=h_{k}-\vartheta s_{k}, k=1, \ldots, N
$$

(see Giovangigli [11, Chapter 2, Section 2.6.1]). In formula (2.2), the symbol $s$ denotes the specific entropy and, as a matter of fact, (2.2) may be viewed as its definition. Furthermore, the entropies $s_{k}, k=1, \ldots, N$, are taken to be constant.

2.1. Equations of states.

[E1] Motivated by Klein et al. [14, we consider that the pressure $p=p(\varrho, \vartheta)$ satisfies the thermal equation of state

$$
p=p_{F}+p_{R}, p_{R}=\frac{a}{3} \vartheta^{4}, a>0,
$$

where $p_{R}$ denotes the radiation pressure, which is particularly relevant in the high temperature regimes typical for combustion processes (see Bose [1, Chapter 11, Section 11.1]). Furthermore, we shall assume that $p_{F}=p_{M}+p_{E}$, where $p_{M}$ is the classical molecular pressure obeying Boyle's law, while $p_{E}$ is the pressure of the electron gas constituent behaving like a Fermi gas in the degenerate regime of high densities and/or low temperatures (see Chapters 1,15 in Eliezer et al. [3]). Thus necessarily $p_{F}$ takes the form

$$
p_{F}=\vartheta^{\frac{5}{2}} P\left(\frac{\varrho}{\vartheta^{\frac{3}{2}}}\right)
$$


where $P \in C^{1}[0, \infty)$ satisfies

$$
\left\{\begin{array}{l}
P(0)=0, P^{\prime}(z)>0 \text { for all } z \geq 0 \\
0<\underline{c}_{v} \leq \frac{\frac{5}{3} P(z)-P^{\prime}(z) z}{z} \leq \bar{c}_{v} \text { for all } z>0 \\
\lim _{z \rightarrow \infty} \frac{P(z)}{z^{\frac{5}{3}}}=p_{\infty}>0 .
\end{array}\right.
$$

Condition (2.6) reflects the fact that the specific heat at constant volume is strictly positive and uniformly bounded. The reader may consult [5] for more details and further discussion.

[E2] The internal energy is given as

$$
e=e_{F}+e_{R}+\sum_{k=1}^{N} h_{k} Y_{k}, \text { with } e_{R}=\frac{a}{\varrho} \vartheta^{4},
$$

where $e_{F}=e_{F}(\varrho, \vartheta), p_{F}(\varrho, \vartheta)$ are interrelated through a general caloric equation of state

$$
p_{F}(\varrho, \vartheta)=\frac{2}{3} \varrho e_{F}(\varrho, \vartheta)
$$

characteristic for mixtures of mono-atomic gases.

[E3] In accordance with (2.2), (2.7) we set

$$
s=s_{F}+s_{R}+\sum_{k=1}^{N} s_{k} Y_{k}, \text { with } s_{R}=\frac{4 a}{3 \varrho} \vartheta^{3}, s_{F}=S\left(\frac{\varrho}{\vartheta^{\frac{3}{2}}}\right),
$$

where

$$
S^{\prime}(z)=-\frac{3}{2} \frac{\frac{5}{3} P(z)-P^{\prime}(z) z}{z^{2}} .
$$

We also suppose the thermodynamic stability

$$
\frac{\partial e(\varrho, \vartheta)}{\partial \vartheta}>0, \frac{\partial p(\varrho, \vartheta)}{\partial \varrho}>0
$$

2.2. Transport coefficients. The transport coefficients satisfy the following.

[C1] We suppose the viscosity coefficients $\mu$ and $\eta$ are continuously differentiable functions of the temperature satisfying

$$
\left\{\begin{array}{l}
0<\underline{\mu}\left(1+\vartheta^{\alpha}\right) \leq \mu(\vartheta) \leq \bar{\mu}\left(1+\vartheta^{\alpha}\right) \\
\left|\mu^{\prime}(\vartheta)\right| \leq M, 0 \leq \eta(\vartheta) \leq \bar{\eta}\left(1+\vartheta^{\alpha}\right)
\end{array}\right.
$$

for all $\vartheta>0$ and for

$$
\frac{2}{5}<\alpha \leq 1
$$

[C2] The species diffusion coefficients coincide for all $k$, specifically,

$$
D_{k}=D \text { for all } k=1, \ldots, n,
$$

where, in accordance with the general axioms postulated in [11, Chapter 7], we assume that $D$ is a continuously differentiable function of $\vartheta$ such that

$$
0<\underline{D} \leq D(\vartheta) \leq \bar{D}\left(1+\vartheta^{\alpha}\right) \text { for all } \vartheta>0 \text {. }
$$


[C3] The heat conductivity is given as the sum

$$
\kappa=\kappa_{F}(\vartheta)+\kappa_{R}(\varrho, \vartheta)
$$

where $\kappa_{F}, \kappa_{R}$ are continuously differentiable functions satisfying

$$
\begin{gathered}
0<\underline{\kappa}_{F} \leq \kappa_{F}(\vartheta) \leq \bar{\kappa}_{F}\left(1+\vartheta^{\alpha}\right) \text { for all } \vartheta>0, \\
0<\underline{\kappa}_{R} \vartheta^{3} \leq \kappa_{R}(\varrho, \vartheta) \leq \bar{\kappa}_{R} \vartheta^{3} \text { for all } \varrho \geq 0, \vartheta>0 .
\end{gathered}
$$

Similarly to the above, the presence of the extra heat conductivity coefficient $\kappa_{R}$ is related to the effect of radiation (see Oxenius [16]).

2.3. Species production rate. The species production rates $\omega_{k}$ are continuous functions of the absolute temperature $\vartheta$ and the species mass fractions $Y_{1}, \ldots, Y_{N}$. For the sake of simplicity, we shall assume that

$$
\left|\bar{\omega}_{k}\left(\vartheta, Y_{1}, \ldots, Y_{N}\right)\right| \leq C_{k}|\vartheta-\bar{\vartheta}| G(\vartheta) \max \left\{Y_{1}, \ldots, Y_{N}\right\} \text { for all } \vartheta \geq 0,0 \leq Y_{k} \leq 1,
$$

where $\bar{\vartheta}=\frac{1}{|\Omega|} \int_{\Omega} \vartheta \mathrm{d} x, C_{k}>0, k=1,2, \ldots, N$, and a smooth nondecreasing function $G$ is defined by

$$
G(\vartheta)=\left\{\begin{array}{l}
0 \quad \text { if } \vartheta \leq \bar{\vartheta}, \\
1 \quad \text { if } \vartheta \rightarrow \infty,
\end{array} \text { and } G(\vartheta)>0 \text { on }(\bar{\vartheta}, \infty)\right.
$$

Notice that we can take $G(\vartheta)=\exp \left(-\frac{1}{\vartheta-\bar{\vartheta}}\right)$ as a typical example. Furthermore, in agreement with the conservation of mass and the second law of thermodynamics, we suppose that

$$
\sum_{k=1}^{N} \omega_{k}=0, \sum_{k=1}^{N} g_{k} \omega_{k} \leq 0
$$

(see Section 3 below).

Finally, we suppose that

$$
\omega_{k}\left(Y_{1}, \ldots Y_{N}\right) \geq 0 \text { whenever } Y_{k}=0 .
$$

\section{Variational formulation.}

3.1. Two initial-boundary-value problems. Let $\Omega \subset \mathbb{R}^{3}$ be a bounded domain with the boundary of class $C^{2+\nu}, \nu>0$. Accordingly, system (1.1)-(1.4) has to be supplemented with a suitable set of boundary conditions in order to obtain, at least formally, a mathematically well-posed problem. The concept of the weak solutions introduced below requires the energy flux through the boundary to be zero. Therefore we suppose that

$$
\left.\mathbf{u} \cdot \mathbf{n}\right|_{\partial \Omega}=0
$$

together with

$$
\left.\mathbf{q} \cdot \mathbf{n}\right|_{\partial \Omega}=\left.\mathcal{F}_{k} \cdot \mathbf{n}\right|_{\partial \Omega}=0,
$$

where $\mathbf{n}$ stands for the outer normal vector. In addition, the impermeability condition (3.1) is supplemented either with the complete slip boundary condition

$$
(\mathbb{S n}) \times\left.\mathbf{n}\right|_{\partial \Omega}=0,
$$

or

$$
\mathbf{u} \times\left.\mathbf{n}\right|_{\partial \Omega}=0
$$


where the latter, combined with (3.2), gives rise to the standard no-slip boundary conditions

$$
\left.\mathbf{u}\right|_{\partial \Omega}=0
$$

\subsection{Variational solutions.}

Definition 3.1. In the spirit of [9] we say that a quantity $\left\{\varrho, \mathbf{u}, \vartheta, Y_{1}, \ldots, Y_{N}\right\}$ is a variational (weak) solution of the Navier-Stokes-Fourier system NSF, respectively, supplemented with the initial data $\left\{\varrho_{0}, \mathbf{u}_{0}, \vartheta_{0}, Y_{1,0}, \ldots, Y_{N, 0}\right\}$ provided that the following hold.

- The density $\varrho$ is a nonnegative function, $\varrho \in L^{\infty}\left(0, T ; L^{\frac{5}{3}}(\Omega)\right)$, the velocity field $\mathbf{u} \in L^{2}\left(0, T ; W_{0}^{1,2}\left(\Omega ; \mathbb{R}^{3}\right)\right)$, and $\varrho \geq 0, \mathbf{u}$ represent a renormalized solution of equation (1.1) on a time-space cylinder $(0, T) \times \Omega$; that is, the integral identity

$$
\int_{0}^{T} \int_{\Omega}\left(\varrho B(\varrho) \partial_{t} \varphi+\varrho B(\varrho) \mathbf{u} \cdot \nabla_{x} \varphi-b(\varrho) \operatorname{div}_{x} \mathbf{u} \varphi\right) \mathrm{d} x \mathrm{~d} t=-\int_{\Omega} \varrho_{0} B\left(\varrho_{0}\right) \varphi(0, \cdot) \mathrm{d} x
$$

holds for any test function $\varphi \in \mathcal{D}([0, T) \times \bar{\Omega})$ and any $b$ such that

$$
b \in L^{\infty} \cap C[0, \infty), B(\varrho)=B(1)+\int_{1}^{\varrho} \frac{b(z)}{z^{2}} \mathrm{~d} z .
$$

- The moment $\varrho \mathbf{u} \in L^{\infty}\left(0, T ; L^{\frac{5}{4}}\left(\Omega ; \mathbb{R}^{3}\right)\right)$, the absolute temperature $\vartheta$ is positive a.e. on the set $(0, T) \times \Omega$,

$$
\vartheta^{\beta} \in L^{2}\left(0, T ; W^{1,2}(\Omega)\right) \text { for all } \beta \in\left[1, \frac{3}{2}\right]
$$

and the balance of momentum holds in the distributional sense, namely

$$
\begin{gathered}
\int_{0}^{T} \int_{\Omega}\left(\varrho \mathbf{u} \cdot \partial_{t} \vec{\varphi}+\varrho \mathbf{u} \otimes \mathbf{u}: \nabla_{x} \vec{\varphi}+\frac{1}{\mathrm{Ma}^{2}} p \operatorname{div}_{x} \vec{\varphi}\right) \mathrm{d} x \mathrm{~d} t= \\
\int_{0}^{T} \int_{\Omega} \mathbb{S}: \nabla_{x} \vec{\varphi} \mathrm{d} x \mathrm{~d} t-\int_{\Omega} \varrho_{0} \mathbf{u}_{0} \cdot \vec{\varphi}(0, \cdot) \mathrm{d} x
\end{gathered}
$$

for any test function $\vec{\varphi} \in \mathcal{D}\left([0, T) ; \mathcal{D}\left(\bar{\Omega} ; \mathbb{R}^{3}\right)\right)$ satisfying $\left.\vec{\varphi} \cdot \mathbf{n}\right|_{\partial \Omega}=0$, or, $\left.\vec{\varphi}\right|_{\partial \Omega}=0$. All quantities appearing in (3.6) are supposed to be at least integrable, and $\mathbb{S}$, $p$ obey the constitutive relations (1.5), (2.5), respectively. In particular, the velocity field $\mathbf{u}$ must belong to a Sobolev space $L^{p}\left(0, T ; W^{1, q}\left(\Omega ; \mathbb{R}^{3}\right)\right)$; therefore it is legitimate to require $\mathbf{u}$ to satisfy the boundary condition (3.4) as the case may be, in the sense of traces.

- The total energy of the system is a constant of motion. Specifically,

$$
\frac{d}{d t} \int_{\Omega}\left(\frac{\mathrm{Ma}^{2}}{2} \varrho|\mathbf{u}|^{2}+\varrho e_{F}(\varrho, \vartheta)+\varrho e_{R}(\varrho, \vartheta)+\sum_{k=1}^{N} \varrho h_{k} Y_{k}\right) \mathrm{d} x=0 .
$$

- The entropy $\varrho s(\varrho, \vartheta) \in L^{\infty}\left(0, T ; L^{1}(\Omega)\right)$, the terms $\varrho s(\varrho, \vartheta) \mathbf{u}, \frac{\kappa(\varrho, \vartheta)}{\vartheta} \nabla_{x} \vartheta$ belong to $L^{1}((0, T) \times \Omega), \log (\vartheta) \in L^{2}\left(0, T ; W^{1,2}(\Omega)\right), Y_{k} \in L^{\infty}((0, T) \times \Omega)$, and the 
integral inequality

$$
\begin{aligned}
& \int_{0}^{T} \int_{\Omega}\left[\varrho s \partial_{t} \varphi+\varrho s \mathbf{u} \cdot \nabla_{x} \varphi+\left(\frac{\mathbf{q}}{\vartheta}-\sum_{k=1}^{N} s_{k} \mathcal{F}_{k}\right) \cdot \nabla_{x} \varphi\right] \mathrm{d} x \mathrm{~d} t \\
& \quad+\int_{0}^{T} \int_{\Omega} \frac{1}{\vartheta}\left(\mathrm{Ma}^{2} \mathbb{S}: \nabla_{x} \mathbf{u}-\frac{\mathbf{q} \cdot \nabla_{x} \vartheta}{\vartheta}-\sum_{k=1}^{N}{\operatorname{Da} \varrho g_{k}}_{\bar{\omega}}\right) \varphi \mathrm{d} x \mathrm{~d} t \\
& \leq-\int_{\Omega} \varrho_{0} s\left(\varrho_{0}, \vartheta_{0}, Y_{1,0}, \ldots, Y_{N, 0}\right) \varphi(0, \cdot) \mathrm{d} x
\end{aligned}
$$

is satisfied for any test function $\varphi \in \mathcal{D}([0, T) \times \bar{\Omega}), \varphi \geq 0$.

Here the quantities $\mathbb{S}, \mathbf{q}$, and $\mathcal{F}_{k}, k=1, \ldots, N$, are given through the constitutive equations (1.5), (1.7), and (1.8), respectively. Moreover, similarly to the above, all quantities must be at least integrable on $(0, T) \times \Omega$. In particular, both $\vartheta$ and $Y_{k}$ belong to a Sobolev space $L^{q}\left(0, T ; W^{1, q}(\Omega)\right.$. In addition, we require $\vartheta(t, x)$ to be positive for a.a. $(t, x) \in(0, T) \times \Omega$ and the entropy production $\sigma$ associated to a weak solution satisfies

$$
\sigma \geq \frac{1}{\vartheta}\left(\mathrm{Ma}^{2} \mathbb{S}: \nabla_{x} \mathbf{u}-\frac{\mathbf{q} \cdot \nabla_{x} \vartheta}{\vartheta}-\sum_{k=1}^{N}{\operatorname{Da} \varrho g_{k}}_{\bar{\omega}}\right) .
$$

- In addition, we introduce a concept of "entropy" solutions for (1.4) requiring the integral identity

$$
\begin{gathered}
-\int_{0}^{T} \int_{\Omega}\left(\varrho Y_{k} \partial_{t} \varphi+\varrho Y_{k} \mathbf{u} \cdot \nabla_{x} \varphi-D \nabla_{x} Y_{k} \cdot \nabla_{x} \varphi\right) \mathrm{d} x \mathrm{~d} t= \\
+\int_{0}^{T} \int_{\Omega} \sum_{k=1}^{N} \operatorname{Da} \varrho \bar{\omega}_{k} \varphi \mathrm{d} x \mathrm{~d} t+\int_{\Omega} \varrho_{0} Y_{k, 0} \varphi(0, \cdot) \mathrm{d} x, k=1, \ldots, N,
\end{gathered}
$$

to be satisfied for any test function $\varphi \in \mathcal{D}([0, T) \times \bar{\Omega})$, together with

$$
\begin{gathered}
-\int_{0}^{T} \partial_{t} \psi \int_{\Omega} \varrho G(\mathbf{Y}) \mathrm{d} x \mathrm{~d} t+\int_{0}^{T} \psi \int_{\Omega} \sum_{k=1}^{N} D \underline{G}\left|\nabla_{x} Y_{k}\right|^{2} \mathrm{~d} x \mathrm{~d} t \leq \\
\int_{0}^{T} \psi \int_{\Omega} \sum_{k=1}^{N} \varrho \frac{\partial G(\mathbf{Y})}{\partial Y_{k}} \omega_{k} \mathrm{~d} x \mathrm{~d} t+\int_{\Omega} \varrho_{0} G\left(\mathbf{Y}_{0}\right) \psi(0) \mathrm{d} x
\end{gathered}
$$

for any $\psi \in \mathcal{D}[0, T), \psi \geq 0$, and any convex $G \in C^{2}\left(R^{d}\right)$,

$$
\sum_{k, l} \frac{\partial^{2} G(\mathbf{Y})}{\partial Y_{k} \partial Y_{l}} \xi_{k} \xi_{l} \geq \underline{G}|\xi|^{2}
$$

where we have set $\mathbf{Y}=\left(Y_{1}, \ldots, Y_{N}\right)$.

In addition,

$$
0 \leq Y_{k} \leq 1 \text { for all } k=1, \ldots, N, \sum_{k=1}^{N} Y_{k}=1 .
$$


The existence of variational solutions for the two initial-boundary-value problems are presented in [9].

We now introduce the weak solutions of the target system (1.14).

Definition 3.2. A trio $\left\{\mathbf{U}, \Theta, Y_{k}\right\}, k=1,2, \ldots, N$, is said to be a variational solution of the system (1.14), supplemented with the boundary conditions

$$
\mathbf{U}=0 \text { or } \mathbf{U} \cdot \mathbf{n}=0, \nabla_{x} Y_{k} \cdot \mathbf{n}=0, k=1,2, \ldots, N, \nabla_{x} \Theta \cdot \mathbf{n}=0
$$

on $\partial \Omega$ and the initial conditions

$$
\mathbf{U}(0, \cdot)=\mathbf{U}_{0}, \Theta(0, \cdot)=\Theta_{0}, Y_{k}(0, \cdot)=Y_{k, 0}, k=1,2, \ldots, N,
$$

if the following conditions hold:

- The velocity vector field $\mathbf{U} \in L^{\infty}\left(0, T ; L^{2}\left(\Omega ; \mathbb{R}^{3}\right)\right) \cap L^{2}\left(0, T ; W_{0}^{1,2}\left(\Omega ; \mathbb{R}^{3}\right)\right)$,

$$
\operatorname{div}_{x} \mathbf{U}=0 \text { a.e. on }(0, T) \times \Omega,
$$

and the integral identity

$$
\begin{aligned}
& \int_{0}^{T} \int_{\Omega}(\left.\bar{\varrho} \mathbf{U} \cdot \partial_{t} \varphi+\bar{\varrho}(\mathbf{U} \otimes \mathbf{U}): \nabla_{x} \varphi\right) \mathrm{d} x \mathrm{~d} t \\
& \quad=\int_{0}^{T} \int_{\Omega} \bar{\mu} \nabla_{x} \mathbf{U}: \nabla_{x} \varphi \mathrm{d} x \mathrm{~d} t-\int_{\Omega} \bar{\varrho} \mathbf{U} \cdot \varphi(0, \cdot) \mathrm{d} x
\end{aligned}
$$

holds for any test function

$$
\varphi \in \mathcal{D}\left((0, T) \times \Omega ; \mathbb{R}^{3}\right), \operatorname{div}_{x} \varphi=0 .
$$

- The temperature $\Theta \in L^{\infty}\left(0, T ; L^{2}\left(\Omega ; \mathbb{R}^{3}\right)\right) \cap L^{2}\left(0, T ; W^{1,2}(\Omega)\right)$, and the integral identity

$$
\begin{aligned}
\int_{0}^{T} \int_{\Omega}\left(\varrho \overline { c } _ { p } \left(\Theta \partial_{t} \varphi\right.\right. & \left.\left.+\Theta \mathbf{U} \cdot \nabla_{x} \varphi\right)-\bar{\kappa} \nabla_{x} \Theta \cdot \nabla_{x} \varphi\right) \mathrm{d} x \mathrm{~d} t \\
& =-\int_{\Omega} \bar{\varrho} \bar{c}_{p} \Theta(0 \varphi(0, \cdot) \mathrm{d} x
\end{aligned}
$$

holds for any test function

$$
\varphi \in \mathcal{D}((0, T) \times \bar{\Omega}) .
$$

- The reactant fraction $Y_{k} \in L^{\infty}((0, T) \times \Omega) \cap L^{2}\left(0, T ; W^{1,2}(\Omega)\right), k=1,2, \ldots, N$, such that

$$
0 \leq Y_{k} \leq 1, \sum_{k=1}^{N} Y_{k}=1, \text { for a.e. }(t, x) \in(0, T) \times \Omega
$$

and the integral identity

$$
\begin{gathered}
\int_{0}^{T} \int_{\Omega}\left(\varrho Y_{k} \partial_{t} \varphi+Y_{k} \mathbf{U} \cdot \nabla_{x} \varphi-D_{k}(\bar{\vartheta}) \nabla_{x} Y_{k} \cdot \nabla_{x} \varphi\right) \mathrm{d} x \mathrm{~d} t \\
=-\int_{\Omega} \bar{\varrho} Y_{k, 0} \varphi(0, \cdot) \mathrm{d} x
\end{gathered}
$$

is satisfied for any test function $\varphi \in \mathcal{D}((0, T) \times \bar{\Omega}))$. 
4. Main results. We now introduce a geometric condition on $\Omega$ which plays a crucial role in the study of the propagation of acoustic waves. Let us consider the following problem:

$$
-\Delta \phi=\lambda \phi \text { in } \Omega, \frac{\partial \phi}{\partial \mathbf{n}}=0 \text { on } \partial \Omega,
$$

where $\phi$ is constant on $\partial \Omega$. We call a solution of the problem (4.1) trivial if $\lambda=0$ and $\phi$ is constant. We also define that $\Omega$ satisfies the suitable assumption if all solutions of the problem (4.1) are trivial. Notice that Schiffer's conjecture shows that every $\Omega$ satisfies the suitable assumption except for the ball, and Feireisl, Novotný [6] give an example of a domain $\Omega$ which is trivial. In two-dimensional space, it is proven that every bounded, simply connected open domain $\Omega \subset \mathbb{R}^{2}$ whose boundary is Lipschitz but not real analytic satisfies the suitable assumption.

4.1. Result on bounded domains. In this section we present the result on bounded domains.

Theorem 4.1. Let $\Omega \subset \mathbb{R}^{3}$ be a bounded domain with a boundary of class $C^{2+\nu}, \nu>0$ and verify the suitable assumption in problem (4.1). Let us assume $\mathrm{Ma}=\epsilon, \mathrm{Da}=\frac{1}{\epsilon}$ and $\left\{\varrho_{\epsilon}, \mathbf{u}_{\epsilon}, \vartheta_{\epsilon}, Y_{k}^{\epsilon}\right\}, k=1,2, \ldots, N$, is a family of variational solutions to the NSF system in the sense of Definition 3.1 with the boundary conditions (3.2) and (3.4). Let us assume that the pressure $p$, the specific internal energy $e$, and the specific entropy $s$ are functions of the state variables $\varrho_{\epsilon}, \vartheta_{\epsilon}, Y_{k}^{\epsilon}, k=1,2, \ldots, N$, satisfying Gibbs' equation (2.2) supplemented with the structural hypotheses (2.4)-(2.10). In addition, suppose that the transport coefficients $\mu, \eta, D$, and $\kappa$ satisfy (2.12) (2.18). Finally, let $\omega_{k}, k=1, \ldots, N$, obey (2.19), (2.21), with the $g_{k}$ given through (2.3). Assume the initial condition as follows:

$$
\varrho_{\epsilon}(0, \cdot)=\varrho_{\epsilon, 0}=\bar{\varrho}+\epsilon \varrho_{\epsilon, 0}^{(1)}, \mathbf{u}_{\epsilon}(0, \cdot)=\mathbf{u}_{\epsilon, 0}, \vartheta(0, \cdot)=\bar{\vartheta}+\epsilon \vartheta_{\epsilon, 0}^{(1)}
$$

and

where

$$
Y_{k}^{\epsilon}(0, \cdot)=\epsilon Y_{k,(1)}^{\epsilon, 0}, k=1,2, \ldots, N
$$

$$
\bar{\varrho}=\frac{1}{|\Omega|} \int_{\Omega} \varrho_{\epsilon, 0} d x, \bar{\vartheta}=\frac{1}{|\Omega|} \int_{\Omega} \vartheta_{\epsilon, 0} d x, \sum_{k=1}^{N} Y_{k,(1)}^{\epsilon, 0}=1,0 \leq Y_{k,(1)}^{\epsilon, 0} \leq 1
$$

and

$$
\varrho_{\epsilon, 0} \rightarrow \varrho_{0}^{(1)}, \mathbf{u}_{\epsilon, 0} \rightarrow \mathbf{u}_{0}^{(1)}, \vartheta_{\epsilon, 0} \rightarrow \vartheta_{0}^{(1)}, Y_{k,(1)}^{\epsilon, 0} \rightarrow Y_{k,(1)}^{0}
$$

as $\epsilon$ tends to 0 , where we have used weakly-* convergence in $L^{\infty}(\Omega)$.

Then, up to a subsequence,

$$
\left\{\begin{array}{l}
\varrho_{\epsilon} \rightarrow \bar{\varrho} \text { in } C\left([0, T] ; L^{1}(\Omega)\right) \cap L^{\infty}\left(0, T ; L^{\frac{5}{3}}(\Omega)\right), \\
\vartheta_{\epsilon} \rightarrow \bar{\vartheta} \text { in } L^{2}\left(0, T ; W^{1,2}(\Omega)\right), \\
\mathbf{u}_{\epsilon} \rightarrow \mathbf{U} \text { strongly in } L^{2}\left(0, T ; L^{2}\left(\Omega ; \mathbb{R}^{3}\right)\right), \\
\frac{Y_{k}^{\epsilon}}{\epsilon} \rightarrow Y_{k} \text { weakly in } L^{2}\left(0, T ; W^{1,2}(\Omega)\right) \text { and weakly }-* \text { in } L^{\infty}((0, T) \times \Omega),
\end{array}\right.
$$


and

$$
\left\{\begin{array}{l}
\varrho_{\epsilon}^{(1)}=\frac{\varrho_{\epsilon}-\bar{\varrho}}{\epsilon} \rightarrow \varrho^{(1)} \text { weakly- } * \text { in } L^{\infty}\left(0, T ; L^{\frac{5}{3}}(\Omega)\right) \\
\vartheta_{\epsilon}^{(1)}=\frac{\vartheta_{\epsilon}-\bar{\vartheta}}{\epsilon} \rightarrow \Theta \text { weakly in } L^{2}\left(0, T ; W^{1,2}(\Omega)\right)
\end{array}\right.
$$

where the trio $\left\{\mathbf{U}, \Theta, Y_{k}\right\}, k=1,2, \ldots, N$, solves the NSF system in the sense of Definition 3.2 with the boundary condition $\left.\mathbf{u}\right|_{\partial \Omega}=0$ and the initial data

$$
\mathbf{U}(0)=\mathbf{H}\left[\mathbf{U}_{0}\right], \quad \Theta(0, j):=\Theta_{0}=\bar{\vartheta} \bar{c}_{p}^{-1}\left(\frac{\partial s(\bar{\varrho}, \bar{\vartheta})}{\varrho} \varrho_{0}^{(1)}+\frac{\partial s(\bar{\varrho}, \bar{\vartheta})}{\vartheta} \vartheta_{0}^{(1)}\right), \quad Y_{k}^{0}=Y_{k,(1)}^{0}
$$

where the Helmholtz projection $\mathbf{H}$ is defined in (4.9):

$$
\mathbf{H}=\mathbf{I}-\mathbf{H}^{\perp}, \mathbf{H}^{\perp}=\nabla \Delta^{-1} \operatorname{div} .
$$

4.2. Result on unbounded domains. We next study incompressible limit problems for multicomponent reactive flows on unbounded domains. Consider an unbounded domain $\Omega \subset \mathbb{R}^{3}$ with a compact regular boundary $\partial \Omega$ and a family of bounded domains $\left\{\Omega_{\epsilon}\right\}_{\epsilon>0}$ satisfying:

$$
\Omega_{\epsilon} \subset \Omega, \partial \Omega \subset \partial \Omega_{\epsilon}, \epsilon \operatorname{dist}\left[x, \partial \Omega_{\epsilon}\right] \rightarrow \infty \text { as } \epsilon \rightarrow 0,
$$

for any $x \in \Omega$. We consider a variational solution to the NSF system in the sense of Definition 3.1 on $\Omega_{\epsilon}$. Then the second main result is the following.

THEOREM 4.2. Let $\Omega \subset \mathbb{R}^{3}$ be an unbounded domain with a compact boundary $\partial \Omega$ of class $C^{2+\nu}, \nu>0$ and a family of bounded domains $\left\{\Omega_{\epsilon}\right\}_{\epsilon>0}$ satisfying (4.10). Let us assume $\mathrm{Ma}=\epsilon, \mathrm{Da}=\frac{1}{\epsilon}$ and $\left\{\varrho_{\epsilon}, \mathbf{u}_{\epsilon}, \vartheta_{\epsilon}, Y_{k}^{\epsilon}\right\}, k=1,2, \ldots, N$, is a family of variational solutions on $(0, T) \times \Omega_{\epsilon}$ to the NSF system in the sense of Definition 3.1 on $\Omega_{\epsilon}$ with the same initial conditions given in Theorem 4.1 and the boundary conditions (3.1), (3.2), and (3.3) on $\Omega_{\epsilon}$. Let us assume that all of the hypotheses in Theorem 4.1 hold. Then we have the same convergence of $\left\{\varrho_{\epsilon}, \mathbf{u}_{\epsilon}, \vartheta_{\epsilon}, Y_{k}^{\epsilon}\right\}$ on any compact $K \subset \Omega$ as given in Theorem 4.1 such that the limits $\left\{\mathbf{U}, \Theta, Y_{k}\right\}$ of $\left\{\mathbf{u}_{\epsilon}, \frac{\vartheta_{\epsilon}-\bar{\vartheta}}{\epsilon}, Y_{k}^{\epsilon}\right\}, k=1,2, \ldots, N$, solve the NSF system in the sense of Definition 3.2 with the no-slip boundary condition $\left.\mathbf{U} \cdot \mathbf{n}\right|_{\partial \Omega}=0$ and the initial data (4.8).

\section{Low Mach Number on the bounded domain with Dirichlet boundary.}

5.1. Energy inequality and uniform bounds. In this section we are going to derive some estimates on the sequence $\left\{\varrho_{\epsilon}, \mathbf{u}_{\epsilon}, \vartheta_{\epsilon}\right\}_{\epsilon>0}$. From the assumption (4.2), we first deduce

$$
\int_{\Omega} \varrho_{\epsilon}(t) \mathrm{d} x=\bar{\varrho}|\Omega|, \quad \int_{\Omega} \varrho_{\epsilon}^{(1)}(t) \mathrm{d} x=0
$$


We now use the total energy balance (3.7) and the entropy inequality (3.8) in order to derive the dissipation equality

$$
\begin{gathered}
\int_{\Omega}\left[\frac{1}{2} \varrho_{\epsilon}\left|\mathbf{u}_{\epsilon}\right|^{2}+\frac{1}{\epsilon^{2}}\left(H_{\bar{\vartheta}}\left(\varrho_{\epsilon}, \vartheta_{\epsilon}\right)-\left(\varrho_{\epsilon}-\bar{\varrho}\right) \frac{\partial H_{\bar{\vartheta}}(\bar{\varrho}, \bar{\vartheta})}{\partial \varrho}-H_{\bar{\vartheta}}(\bar{\varrho}, \bar{\vartheta})\right)\right](t) \mathrm{d} x \\
+\frac{\bar{\vartheta}}{\epsilon^{2}} \sigma_{\epsilon}[[0, t] \times \bar{\Omega}]=\int_{\Omega} \frac{1}{2} \varrho_{\epsilon, 0}\left|\mathbf{u}_{\epsilon, 0}\right|^{2} \mathrm{~d} x \\
+\int_{\Omega} \frac{1}{\epsilon^{2}}\left(H_{\bar{\vartheta}}\left(\varrho_{\epsilon, 0}, \vartheta_{\epsilon, 0}\right)-\left(\varrho_{\epsilon, 0}-\bar{\varrho}\right) \frac{\partial H_{\bar{\vartheta}}(\bar{\varrho}, \bar{\vartheta})}{\partial \varrho}-H_{\bar{\vartheta}}(\bar{\varrho}, \bar{\vartheta})\right)(t) \mathrm{d} x \\
-\int_{\Omega} \frac{1}{\epsilon^{2}} \sum_{k=1}^{N}\left(h_{k}-\bar{\vartheta} s_{k}\right)\left(\varrho_{\epsilon} Y_{k}^{\epsilon}-\varrho_{\epsilon, 0} Y_{0}^{\epsilon}\right) \mathrm{d} x
\end{gathered}
$$

for a.e. $t \in(0, T)$, where the Helmholtz function $H_{\bar{\vartheta}}$ is defined by

$$
H_{\bar{\vartheta}}(\bar{\varrho}, \bar{\vartheta})=\bar{\varrho} \bar{e}(\bar{\varrho}, \bar{\vartheta})-\bar{\vartheta} \bar{\varrho} \bar{s}(\bar{\varrho}, \bar{\vartheta}),
$$

and where we set $\bar{e}(\bar{\varrho}, \bar{\vartheta}):=e_{R}(\bar{\varrho}, \bar{\vartheta})+e_{F}(\bar{\varrho}, \bar{\vartheta})$ and $\bar{s}(\bar{\varrho}, \bar{\vartheta}):=s_{R}(\bar{\varrho}, \bar{\vartheta})+s_{F}(\bar{\varrho}, \bar{\vartheta})$.

At this point we introduce the set of essential values $\mathcal{O}_{\text {ess }} \subset(0, \infty)^{2}$,

$$
\mathcal{O}_{\text {ess }}:=\left\{(\varrho, \vartheta) \in R^{2} \mid \varrho / 2<\varrho<2 \bar{\varrho}, \bar{\vartheta}<\varrho<2 \bar{\vartheta}\right\}
$$

and the residual set

$$
\mathcal{O}_{\text {res }}:=(0, \infty)^{2} \cap \mathcal{O}_{\text {ess }}^{c} .
$$

We next define the essential set and residual set of points $(t, x) \times \Omega$ as follows:

$$
\begin{gathered}
\mathcal{M}_{\mathrm{ess}}^{\epsilon} \subset(0, T) \times \Omega, \\
\mathcal{M}_{\mathrm{ess}}^{\epsilon}=\left\{(t, x) \in(0, T) \times \Omega \mid\left(\varrho_{\epsilon}(t, x), \vartheta_{\epsilon}(t, x)\right) \in \mathcal{O}_{\mathrm{ess}}\right\}, \\
\mathcal{M}_{\mathrm{res}}^{\epsilon}=((0, T) \times \Omega) \cap\left(\mathcal{M}_{\mathrm{ess}}^{\epsilon}\right)^{c} .
\end{gathered}
$$

Finally, each measurable function $g$ can be decomposed as

$$
g=[g]_{\mathrm{ess}}+[g]_{\mathrm{res}}
$$

and we set

$$
[g]_{\mathrm{ess}}=g 1_{\mathcal{M}_{\mathrm{ess}}^{\epsilon}},[g]_{\mathrm{res}}=g 1_{\mathcal{M}_{\mathrm{res}}^{\epsilon}}=g-[g]_{\mathrm{ess}} .
$$

In order to exploit relation (5.2), we need to investigate the structural properties of the Helmholtz function $H_{\bar{\vartheta}}$. More precisely, we show that the quantity

$$
H_{\bar{\vartheta}}\left(\varrho_{\epsilon}, \vartheta_{\epsilon}\right)-\left(\varrho_{\epsilon}-\bar{\varrho}\right) \frac{\partial H_{\bar{\vartheta}}(\bar{\varrho}, \bar{\vartheta})}{\partial \varrho}-H_{\bar{\vartheta}}(\bar{\varrho}, \bar{\vartheta})
$$

is nonnegative and strictly coercive, attaining its global minimum zero at $(\bar{\varrho}, \bar{\vartheta})$. The structural properties of the Helmholtz function $H_{\bar{\vartheta}}$ are as follows. 
Lemma 5.1. Let $H_{\bar{\vartheta}}(\varrho, \vartheta)$ be the Helmholtz function defined in (5.3) and $\bar{\varrho}>0, \bar{\vartheta}>0$ be constants. Let $\mathcal{O}_{\text {ess }}, \mathcal{O}_{\text {res }}$ be the sets of essential and residual values in (5.4) and (5.5). Then there exist $c_{i}=c_{i}(\bar{\varrho}, \bar{\vartheta}), i=1, \ldots, 4$, such that

1.

$$
\begin{aligned}
c_{1}\left(|\varrho-\bar{\varrho}|^{2}+|\vartheta-\bar{\vartheta}|^{2}\right) & \leq H_{\bar{\vartheta}}(\varrho, \vartheta)-(\varrho-\bar{\varrho}) \frac{\partial H_{\bar{\vartheta}}(\bar{\varrho}, \bar{\vartheta})}{\partial \varrho}-H_{\bar{\vartheta}}(\bar{\varrho}, \bar{\vartheta}) \\
& \leq c_{1}\left(|\varrho-\bar{\varrho}|^{2}+|\vartheta-\bar{\vartheta}|^{2}\right)
\end{aligned}
$$

for all $(\varrho, \vartheta) \in \mathcal{O}_{\text {ess }}$.

2.

$$
\begin{aligned}
H_{\bar{\vartheta}}(\varrho, \vartheta) & -(\varrho-\bar{\varrho}) \frac{\partial H_{\bar{\vartheta}}(\bar{\varrho}, \bar{\vartheta})}{\partial \varrho}-H_{\bar{\vartheta}}(\bar{\varrho}, \bar{\vartheta}) \\
& \geq \inf _{(r, \Theta) \in \partial \mathcal{O}_{\text {ess }}}\left\{H_{\bar{\vartheta}}(r, \Theta)-(r-\bar{\varrho}) \frac{\partial H_{\bar{\vartheta}}(\bar{\varrho}, \bar{\vartheta})}{\partial \varrho}-H_{\bar{\vartheta}}(\bar{\varrho}, \bar{\vartheta})\right\} \\
& =c_{3}(\bar{\varrho}, \bar{\vartheta})>0
\end{aligned}
$$

for all $(\varrho, \vartheta) \in \mathcal{O}_{\text {res }}$.

3.

$$
\begin{aligned}
H_{\bar{\vartheta}}(\varrho, \vartheta) & -(\varrho-\bar{\varrho}) \frac{\partial H_{\bar{\vartheta}}(\bar{\varrho}, \bar{\vartheta})}{\partial \varrho}-H_{\bar{\vartheta}}(\bar{\varrho}, \bar{\vartheta}) \\
& \geq c_{4} \varrho \bar{e}(\varrho, \vartheta)+\varrho|\bar{s}(\varrho, \vartheta)|
\end{aligned}
$$

for all $(\varrho, \vartheta) \in \mathcal{O}_{\text {res }}$.

The proof of Lemma 5.1 is provided in [6]. By virtue of the inequality (5.9), one can easily deduce

$$
\begin{aligned}
& \left\|\left\{\frac{\varrho_{\epsilon}-\bar{\varrho}}{\epsilon}\right\}_{\mathrm{ess}}(t)\right\|_{L^{2}(\Omega)}^{2}+\left\|\left\{\frac{\vartheta_{\epsilon}-\bar{\vartheta}}{\epsilon}\right\}_{\mathrm{ess}}(t)\right\|_{L^{2}(\Omega)}^{2} \\
& \leq \frac{C}{\epsilon^{2}} \int_{\Omega}\left(H_{\bar{\vartheta}}\left(\varrho_{\epsilon}, \vartheta_{\epsilon}\right)-\left(\varrho_{\epsilon}-\bar{\varrho}\right) \frac{\partial H_{\bar{\vartheta}}(\bar{\varrho}, \bar{\vartheta})}{\partial \varrho}-H_{\bar{\vartheta}}(\bar{\varrho}, \bar{\vartheta})\right)(t) \mathrm{d} x .
\end{aligned}
$$

Dynamics of viscous compressible fluids. By virtue of (2.4), (2.7), and (2.9) together with (5.11), it follows that

$$
\begin{aligned}
\left\|\left\{\varrho_{\epsilon}\right\}_{\mathrm{res}}(t)\right\|_{L^{\frac{5}{3}(\Omega)}}^{\frac{5}{3}}+\left\|\left\{\vartheta_{\epsilon}\right\}_{\mathrm{res}}(t)\right\|_{L^{4}(\Omega)}^{4}+\left\|\left\{\varrho_{\epsilon} \bar{s}\left(\varrho_{\epsilon}, \vartheta_{\epsilon}\right)\right\}_{\mathrm{res}}(t)\right\|_{L^{1}(\Omega)} \\
\leq \frac{C}{\epsilon^{2}} \int_{\Omega}\left(H_{\bar{\vartheta}}\left(\varrho_{\epsilon}, \vartheta_{\epsilon}\right)-\left(\varrho_{\epsilon}-\bar{\varrho}\right) \frac{\partial H_{\bar{\vartheta}}(\bar{\varrho}, \bar{\vartheta})}{\partial \varrho}-H_{\bar{\vartheta}}(\bar{\varrho}, \bar{\vartheta})\right)(t) \mathrm{d} x
\end{aligned}
$$

for a.e. $t \in(0, T)$. 
We now control the last term on the dissipation inequality (5.2). Following the entropy equation (3.8), we get

$$
\begin{aligned}
\sum_{k=1}^{N} \frac{h_{k}-\bar{\vartheta} s_{k}}{\epsilon^{2}} \int_{\Omega}\left(\varrho_{\epsilon} Y_{k}^{\epsilon}(t)-\varrho_{\epsilon, 0} Y_{k, 0}^{\epsilon}\right) \mathrm{d} x \\
\quad=\sum_{k=1}^{N} \frac{h_{k}-\bar{\vartheta} s_{k}}{\epsilon^{3}} \int_{\Omega} \varrho_{\epsilon} \bar{\omega}_{k}\left(\vartheta_{\epsilon}, Y_{1}^{\epsilon}, \ldots, Y_{N}^{\epsilon}\right) \mathrm{d} x
\end{aligned}
$$

and thus the equality (5.14) yields

$$
\begin{aligned}
& -\sum_{k=1}^{N} \frac{g_{k}}{\epsilon^{2}} \int_{\Omega}\left(\varrho_{\epsilon} Y_{k}^{\epsilon}(t)-\varrho_{\epsilon, 0} Y_{k, 0}^{\epsilon}\right) \mathrm{d} x \\
& =-\sum_{k=1}^{N} \frac{g_{k}}{\epsilon^{2}} \int_{\Omega}\left(\varrho_{\epsilon} Y_{k}^{\epsilon}(t)-\varrho_{\epsilon, 0} Y_{k, 0}^{\epsilon}\right) \mathrm{d} x \\
& +\sum_{k=1}^{N} \frac{g_{k}}{\epsilon^{3}} \int_{0}^{t} \int_{\Omega} \frac{\bar{\vartheta}}{\vartheta_{\epsilon}} \varrho_{\epsilon} \bar{\omega}_{k}\left(\vartheta_{\epsilon}, Y_{1}^{\epsilon}, \ldots, Y_{N}^{\epsilon}\right) \mathrm{d} x \mathrm{~d} t \\
& -\sum_{k=1}^{N} \frac{g_{k}}{\epsilon^{3}} \int_{0}^{t} \int_{\Omega} \frac{\bar{\vartheta}}{\vartheta_{\epsilon}} \varrho_{\epsilon} \bar{\omega}_{k}\left(\vartheta_{\epsilon}, Y_{1}^{\epsilon}, \ldots, Y_{N}^{\epsilon}\right) \mathrm{d} x \mathrm{~d} t \\
& =\sum_{k=1}^{N}\left(g_{k}\right) \int_{0}^{t} \int_{\Omega}\left(\frac{\bar{\vartheta}-\vartheta_{\epsilon}}{\epsilon}\right)\left(\frac{\varrho_{\epsilon}}{\vartheta_{\epsilon}}\right)\left(\frac{\bar{\omega}_{k}\left(\bar{\vartheta}, Y_{1}^{\epsilon}, \ldots, Y_{N}^{\epsilon}\right)-\bar{\omega}_{k}\left(\vartheta_{\epsilon}, Y_{1}^{\epsilon}, \ldots, Y_{N}^{\epsilon}\right)}{\epsilon^{2}}\right) \mathrm{d} x \mathrm{~d} t \\
& -\sum_{k=1}^{N} \frac{g_{k}}{\epsilon^{3}} \int_{0}^{t} \int_{\Omega} \frac{\bar{\vartheta}}{\vartheta_{\epsilon}} \varrho_{\epsilon} \bar{\omega}_{k}\left(\vartheta_{\epsilon}, Y_{1}^{\epsilon}, \ldots, Y_{N}^{\epsilon}\right) \mathrm{d} x \mathrm{~d} t \\
& \leq C\left(Y_{1,0}^{\epsilon,(1)}, \ldots, Y_{N, 0}^{\epsilon,(1)}\right)\left(\int_{0}^{t}\left\|\left\{\frac{\vartheta_{\epsilon}-\bar{\vartheta}}{\epsilon}\right\}_{\mathrm{ess}}(t)\right\|_{L^{2}(\Omega)}^{2} \mathrm{~d} t\right. \\
& +\frac{1}{\epsilon^{2}} \int_{0}^{t} \int_{\Omega}\left(\left\{\vartheta_{\epsilon}\right\}_{\text {res }}^{4}+\left\{\varrho_{\epsilon}\right\}_{\text {res }}^{\frac{5}{3}}\right) \mathrm{d} x \mathrm{~d} t \\
& -\sum_{k=1}^{N} \frac{g_{k}}{\epsilon^{3}} \int_{0}^{t} \int_{\Omega} \frac{\bar{\vartheta}}{\vartheta_{\epsilon}} \varrho_{\epsilon} \bar{\omega}_{k}\left(\vartheta_{\epsilon}, Y_{1}^{\epsilon}, \ldots, Y_{N}^{\epsilon}\right) \mathrm{d} x \mathrm{~d} t,
\end{aligned}
$$

where we have used the maximum principle for the equation (3.17) and

$$
0 \leq \varrho_{\epsilon}(t, x) \frac{Y_{k}^{\epsilon}(t, x)}{\epsilon} \leq \varrho_{\epsilon}(t, x) \underset{x \in \Omega}{\operatorname{ess} \sup _{k, 0}} Y_{k,(1)}^{\epsilon}(y), k=1,2, \ldots, N,
$$

for almost every $(t, x) \in(0, T) \times \Omega$. 
Gronwall's inequality applied to (5.2) together with (5.12), (5.13), (5.15) yields

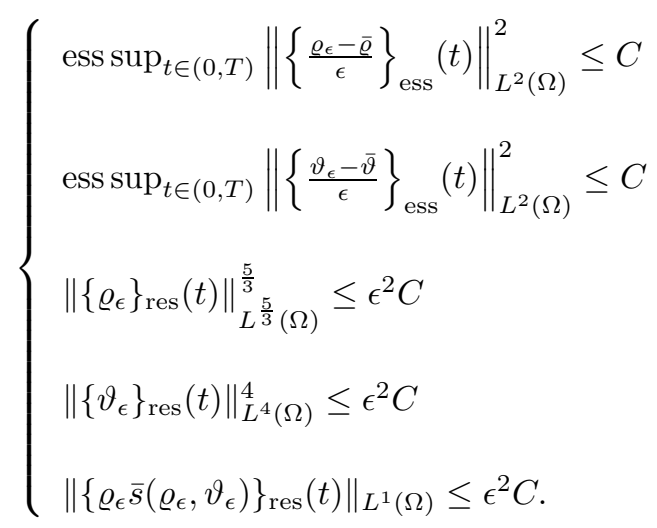

In addition, we get

$$
\left\{\begin{array}{l}
\underset{t \in(0, T)}{\operatorname{ess} \sup }\left\|\sqrt{\varrho_{\epsilon}} \mathbf{u}_{\epsilon}(t)\right\|_{L^{2}\left(\Omega ; R^{3}\right)} \leq C \\
\left\|\frac{\sigma_{\epsilon}}{\epsilon^{2}}+\sum_{k=1}^{N} \frac{g_{k}}{\epsilon^{3}} \int_{0}^{t} \int_{\Omega} \frac{\bar{\vartheta}}{\vartheta_{\epsilon}} \varrho_{\epsilon} \bar{\omega}_{k}\left(\vartheta_{\epsilon}, Y_{1}^{\epsilon}, \ldots, Y_{N}^{\epsilon}\right)\right\|_{\mathcal{M}^{+}([0, T] \times \Omega)} \leq C \\
\left\|\nabla_{x} \mathbf{u}_{\epsilon}+\mathbf{u}_{\epsilon}^{t}-\frac{2}{3} \operatorname{div}_{x} \mathbf{u}_{\epsilon} \mathbb{I}\right\|_{L^{2}((0, T) \times \Omega)} \leq C \\
\left\|\nabla_{x} \vartheta_{\epsilon}\right\|_{L^{2}((0, T) \times \Omega)} \leq \epsilon^{2} C \\
\left\|\nabla_{x} \log \left(\vartheta_{\epsilon}\right)\right\|_{L^{2}((0, T) \times \Omega)} \leq \epsilon^{2} C,
\end{array}\right.
$$

where $g_{k}=h_{k}-\bar{\vartheta} s_{k}, k=1,2, \ldots, N$. As a direct consequence of (15.10), it follows that

$$
\underset{t \in(0, T)}{\operatorname{ess} \sup }\left|\mathcal{M}_{\text {res }}^{\epsilon}[t]\right| \leq \epsilon^{2} C \text {. }
$$

We also use the following Korn-Poincaré inequality to obtain

$$
\left\{\mathbf{u}_{\epsilon}\right\}_{\epsilon>0} \text { bounded in } L^{2}\left(0, T ; W^{1,2}\left(\Omega ; \mathbb{R}^{3}\right)\right) .
$$

Proposition 5.2. Let $\Omega \subset \mathbb{R}^{3}$ be a bounded Lipschitz domain. Assume that $r$ is a nonnegative function such that

$$
0<C_{1} \leq \int_{\Omega} r \mathrm{~d} x, \int_{\Omega} r^{\alpha} \mathrm{d} x \leq C \text { for a certain } \alpha>1 .
$$

Then

$$
\|\mathbf{v}\|_{W^{1, p}\left(\Omega ; \mathbb{R}^{3}\right)} \leq C\left(p, C_{1}, C_{2}\right)\left(\left\|\nabla_{x} \mathbf{v}+\nabla_{x}^{\perp} \mathbf{v}-\frac{2}{3} \operatorname{div}_{x} \mathbf{v}\right\|_{L^{p}\left(\Omega ; R^{3}\right)}+\int_{\Omega} r|\mathbf{v}| \mathrm{d} x\right.
$$

for any $\mathbf{v} \in W^{1, p}\left(\Omega ; \mathbb{R}^{3}\right), 1<p<\infty$. 
The equality (3.17) together with (5.16) is able to give rise to the following uniform estimate:

which implies that

$$
\left\|\frac{Y_{k}^{\epsilon}}{\epsilon}\right\|_{L^{2}\left(0, T ; W^{1,2}(\Omega)\right)} \leq C
$$

$$
\frac{1}{\epsilon} \nabla_{x} Y_{k}^{\epsilon} \rightarrow \nabla_{x} Y_{k} \text { weakly in } L^{2}\left(0, T ; L^{2}(\Omega)\right) .
$$

5.2. Convergence of continuity equation. We will use the uniform estimate (5.20) to deduce

$$
\mathbf{u}_{\epsilon} \rightarrow \mathbf{U} \text { weakly in } L^{2}\left(0, T ; W^{1,2}\left(\Omega ; \mathbb{R}^{3}\right)\right)
$$

up to a subsequence of $\{\epsilon>0\}$. In accordance with (5.17), we have

$$
\left[\frac{\varrho_{\epsilon}-\bar{\varrho}}{\epsilon}\right]_{\mathrm{ess}} \rightarrow \varrho^{(1)} \text { weakly }-* \text { in } L^{\infty}\left(0, T ; L^{2}(\Omega)\right),
$$

and

$$
\left[\frac{\varrho_{\epsilon}-\bar{\varrho}}{\epsilon}\right]_{\mathrm{res}} \rightarrow 0 \text { in } L^{\infty}\left(0, T ; L^{\frac{5}{3}}(\Omega)\right)
$$

whence

$$
\left[\frac{\varrho_{\epsilon}-\bar{\varrho}}{\epsilon}\right] \rightarrow \varrho^{(1)} \text { weakly- } * \text { in } L^{\infty}\left(0, T ; L^{\frac{5}{3}}(\Omega)\right),
$$

and thus we obtain

$$
\varrho_{\epsilon} \rightarrow \bar{\varrho} \text { in } L^{\infty}\left(0, T ; L^{\frac{5}{3}}(\Omega)\right),
$$

and so we can take the limit of $\epsilon$ in the continuity equation (3.15) to get

$$
\int_{0}^{T} \int_{\Omega} \mathbf{U} \cdot \nabla_{x} \varphi \mathrm{d} x \mathrm{~d} t=0
$$

for all $\varphi \in C_{c}^{\infty}((0, T) \times \Omega)$.

5.3. Convergence of entropy balance. In this section we will first use the uniform estimates established in the previous section in order to check the convergence in the equations (3.16) and to identify the limit problem. To do this, we need the general result and we can see the details of the proof in [6].

Proposition 5.3. Let $\left\{\varrho_{\epsilon}\right\}_{\epsilon>0},\left\{\vartheta_{\epsilon}\right\}_{\epsilon>0}$ be two sequences of nonnegative measurable functions such that

$$
\left[\varrho_{\epsilon}^{(1)}\right]_{\mathrm{ess}} \rightarrow \varrho^{(1)},\left[\vartheta_{\epsilon}^{(1)}\right]_{\mathrm{ess}} \rightarrow \vartheta^{(1)},
$$

where the convergence means the weakly-* limit in $L^{\infty}\left(0, T ; L^{2}(\Omega)\right)$ as $\epsilon \rightarrow 0$ and

$$
\varrho_{\epsilon}^{(1)}=\frac{\varrho_{\epsilon}-\bar{\varrho}}{\epsilon}, \quad \vartheta_{\epsilon}^{(1)}=\frac{\vartheta_{\epsilon}-\bar{\vartheta}}{\epsilon} .
$$

Suppose that

$$
\underset{t \in(0, T)}{\operatorname{ess} \sup _{\text {res }}}\left|\mathcal{M}_{\text {re }}^{\epsilon}(t)\right| \leq \epsilon^{2} C .
$$

Let $G \in C^{1}\left(\overline{\mathcal{O}_{\text {ess }}}\right)$ be a given function.

Then,

$$
\frac{\left[G\left(\varrho_{\epsilon}, \vartheta_{\epsilon}\right)\right]_{\mathrm{ess}}-G(\bar{\varrho}, \bar{\vartheta})}{\epsilon} \rightarrow \frac{\partial G(\bar{\varrho}, \bar{\vartheta})}{\partial \varrho} \varrho^{(1)}+\frac{\partial G(\bar{\varrho}, \bar{\vartheta})}{\partial \vartheta} \vartheta^{(1)}
$$


where the convergence is weakly-* in $L^{\infty}\left(0, T ; L^{2}(\Omega)\right)$. Moreover, if $G \in C^{2}\left(\overline{\mathcal{O}_{\text {ess }}}\right)$, then

$$
\begin{gathered}
\left\|\frac{\left[G\left(\varrho_{\epsilon}, \vartheta_{\epsilon}\right)\right]_{\mathrm{ess}}-G(\bar{\varrho}, \bar{\vartheta})}{\epsilon}-\frac{\partial G(\bar{\varrho}, \bar{\vartheta})}{\partial \varrho}\left[\varrho_{\epsilon}^{(1)}\right]_{\mathrm{ess}}-\frac{\partial G(\bar{\varrho}, \bar{\vartheta})}{\partial \vartheta}\left[\vartheta_{\epsilon}^{(1)}\right]_{\mathrm{ess}}\right\|_{L^{\infty}\left(0, T ; L^{1}(\Omega)\right)} \\
\leq \epsilon^{2} C .
\end{gathered}
$$

To begin with this section, we will use the uniform estimate (5.17) and Proposition 5.3 to get

$$
\left[\frac{\vartheta_{\epsilon}-\bar{\vartheta}}{\epsilon}\right]_{\mathrm{ess}} \rightarrow \vartheta^{(1)} \text { weakly- } * \text { in } L^{\infty}\left(0, T ; L^{2}(\Omega)\right)
$$

passing to a suitable subsequence and thus the estimates (5.19) and (5.21) together with Proposition 5.3 yield

$$
\left[\frac{\vartheta_{\epsilon}-\bar{\vartheta}}{\epsilon}\right]_{\mathrm{res}} \rightarrow 0 \text { in } L^{\infty}\left(0, T ; W^{1,2}(\Omega)\right) .
$$

We now investigate the limit problem from the entropy balance equation (3.8) and we rewrite it again:

$$
\begin{gathered}
\int_{0}^{T} \int_{\Omega} \varrho_{\epsilon}\left(\frac{s\left(\varrho_{\epsilon}, \vartheta_{\epsilon}\right)-s(\bar{\varrho}, \bar{\vartheta})}{\epsilon}\right)\left(\partial_{t} \varphi+\mathbf{u}_{\epsilon} \cdot \nabla_{x} \varphi\right) \mathrm{d} t \\
-\int_{0}^{T} \int_{\Omega} \frac{k\left(\varrho_{\epsilon}, \vartheta_{\epsilon}\right)}{\vartheta_{\epsilon}} \cdot \nabla_{x}\left(\frac{\vartheta_{\epsilon}}{\epsilon}\right) \cdot \nabla_{x} \varphi \mathrm{d} t+\frac{1}{\epsilon}\left\langle\sigma_{\epsilon}, \varphi\right\rangle_{[\mathcal{M}, C]([0, T] \times \Omega)} \\
+\int_{0}^{T} \int_{\Omega} \sum_{k=1}^{N} \frac{1}{\epsilon} s_{k} \mathcal{F}_{k} \cdot \nabla_{x} \varphi \mathrm{d} x \mathrm{~d} t \\
=-\int_{\Omega} \varrho_{\epsilon, 0}\left(\frac{s\left(\varrho_{\epsilon, 0}, \vartheta_{\epsilon, 0}, Y_{1,0}^{\epsilon}, \ldots, Y_{N, 0}^{\epsilon}\right)-s\left(\bar{\varrho}, \bar{\vartheta}, Y_{1,0}, \ldots, Y_{N, 0}\right)}{\epsilon}\right) \varphi(0, \cdot) \mathrm{d} x
\end{gathered}
$$

is satisfied for any test function $\varphi \in \mathcal{D}([0, T) \times \bar{\Omega})$.

To identify the limit problem of (5.30), we first control the first part of the entropy balance. We write

$$
\begin{aligned}
& \varrho_{\epsilon}\left(\frac{s\left(\varrho_{\epsilon}, \vartheta_{\epsilon}\right)-s(\bar{\varrho}, \bar{\vartheta})}{\epsilon}\right)=\left[\varrho_{\epsilon}\right]_{\mathrm{ess}} \frac{\left[s\left(\varrho_{\epsilon}, \vartheta_{\epsilon}\right)\right]_{\mathrm{ess}}-s(\bar{\varrho}, \bar{\vartheta})}{\epsilon} \\
& +\left[\frac{\varrho_{\epsilon}}{\epsilon}\right]_{\mathrm{res}}\left(\left[s\left(\varrho_{\epsilon}, \vartheta_{\epsilon}\right)\right]_{\mathrm{ess}}-s(\bar{\varrho}, \bar{\vartheta})\right)+\left[\frac{\varrho_{\epsilon} s\left(\varrho_{\epsilon}, \vartheta_{\epsilon}\right)}{\epsilon}\right]_{\mathrm{res}}
\end{aligned}
$$

Following Proposition 5.3 and (5.27), we can easily see that

$$
\left[\varrho_{\epsilon}\right]_{\mathrm{ess}} \frac{\left[s\left(\varrho_{\epsilon}, \vartheta_{\epsilon}\right)\right]_{\mathrm{ess}}}{\epsilon} \rightarrow \bar{\varrho}\left(\frac{\partial s(\bar{\varrho}, \bar{\vartheta})}{\partial \varrho} \varrho^{(1)}+\frac{\partial s(\bar{\varrho}, \bar{\vartheta})}{\partial \vartheta} \vartheta^{(1)}\right) .
$$

We next investigate the limit of the second line in (5.31). In accordance with the estimate (5.17),

$$
\left[\frac{\varrho_{\epsilon}}{\epsilon}\right]_{\mathrm{res}}\left(\left[s\left(\varrho_{\epsilon}, \vartheta_{\epsilon}\right)\right]_{\mathrm{ess}}-s(\bar{\varrho}, \bar{\vartheta})\right) \rightarrow 0 \text { in } L^{\infty}\left(0, T ; L^{\frac{5}{3}}(\Omega)\right)
$$


By virtue of the structural hypotheses (2.9), we get

$$
\left|\varrho_{\epsilon} s\left(\varrho_{\epsilon}, \vartheta_{\epsilon}\right)\right| \leq C\left(1+\varrho_{\epsilon}\left|\log \left(\varrho_{\epsilon}\right)\right|+\varrho_{\epsilon}\left|\log \left(\vartheta_{\epsilon}\right)-\log (\bar{\vartheta})\right|+\vartheta_{\epsilon}^{3}\right) .
$$

The estimates (5.17) and (5.18) together with (5.2) yield

$$
\left\{\frac{1}{\epsilon}\left(1+\vartheta_{\epsilon}^{3}+\varrho_{\epsilon}\left|\log \left(\varrho_{\epsilon}\right)\right|\right)\right\}_{\text {res }} \rightarrow 0 \text { in } L^{\infty}\left(0, T ; L^{\frac{4}{3}}(\Omega)\right),
$$

which implies that

$$
\left\{\frac{1}{\epsilon}\left(1+\vartheta_{\epsilon}^{3}+\varrho_{\epsilon}\left|\log \left(\varrho_{\epsilon}\right)\right|\right) \mathbf{u}_{\epsilon}\right\}_{\text {res }} \rightarrow 0 \text { in } L^{\infty}\left(0, T ; L^{p}\left(\Omega ; \mathbb{R}^{3}\right)\right) \text { for a certain } p>1 .
$$

We also obtain from the Sobolev imbedding theorem that

$$
\left\{\frac{\varrho_{\epsilon}\left|\log \left(\vartheta_{\epsilon}\right)-\log (\bar{\vartheta})\right|}{\epsilon}\right\}_{\text {res }} \rightarrow 0 \text { in } L^{2}\left(0, T ; L^{p}\left(\Omega ; \mathbb{R}^{3}\right)\right) \text { for a certain } p>1,
$$

and

$$
\left\{\frac{\varrho_{\epsilon}\left|\log \left(\vartheta_{\epsilon}\right)-\log (\bar{\vartheta})\right|}{\epsilon} \mathbf{u}_{\epsilon}\right\}_{\text {res }} \rightarrow 0 \text { in } L^{2}\left(0, T ; L^{q}\left(\Omega ; \mathbb{R}^{3}\right)\right) \text { for a certain } p>1 .
$$

Finally, we apply (5.35), (5.36), (5.37), and (5.38) to (5.34) in order to obtain

$$
\left\{\frac{\varrho_{\epsilon} s\left(\varrho_{\epsilon}, \vartheta_{\epsilon}\right)}{\epsilon}\right\}_{\text {res }} \rightarrow 0 \text { in } L^{2}\left(0, T ; L^{p}\left(\Omega ; \mathbb{R}^{3}\right)\right) \text { for a certain } p>1,
$$

and

$$
\left\{\frac{\varrho_{\epsilon} s\left(\varrho_{\epsilon}, \vartheta_{\epsilon}\right)}{\epsilon} \mathbf{u}_{\epsilon}\right\}_{\text {res }} \rightarrow 0 \text { in } L^{2}\left(0, T ; L^{q}\left(\Omega ; \mathbb{R}^{3}\right)\right) \text { for a certain } p>1 .
$$

Finally, we have to identify the weak limit $\mathbf{D}$ :

$$
\left[\varrho_{\epsilon}\right]_{\mathrm{ess}} \frac{\left[s\left(\varrho_{\epsilon}, \vartheta_{\epsilon}\right)\right]_{\mathrm{ess}}-s(\bar{\varrho}, \bar{\vartheta})}{\epsilon} \mathbf{u}_{\epsilon} \rightarrow \mathbf{D} \text { weakly in } L^{2}\left(0, T ; L^{\frac{3}{2}}\left(\Omega ; R^{3}\right)\right) .
$$

To this end, we need the Div-Curl lemma. Let us set

$$
\begin{aligned}
\mathbf{U}_{\epsilon}=\left[\left[\varrho_{\epsilon}\right]_{\mathrm{ess}} \frac{\left[s\left(\varrho_{\epsilon}, \vartheta_{\epsilon}\right)\right]_{\mathrm{ess}}-s(\bar{\varrho}, \bar{\vartheta})}{\epsilon},\left[\varrho_{\epsilon}\right]_{\mathrm{ess}} \frac{\left[s\left(\varrho_{\epsilon}, \vartheta_{\epsilon}\right)\right]_{\mathrm{ess}}-s(\bar{\varrho}, \bar{\vartheta})}{\epsilon} \mathbf{u}_{\epsilon}\right. \\
\left.-\left[\frac{k\left(\varrho_{\epsilon}, \vartheta_{\epsilon}\right)}{\vartheta_{\epsilon}}\right]_{\mathrm{ess}} \nabla_{x}\left(\frac{\vartheta_{\epsilon}}{\epsilon}\right)\right], \\
\mathbf{V}_{\epsilon}=\left[\mathbf{u}_{\epsilon}, 0,0,0\right] .
\end{aligned}
$$

Using the previous estimates, $\mathbf{U}_{\epsilon}, \mathbf{V}_{\epsilon}$ meet all hypotheses of the Div-Curl lemma and thus we obtain

$$
\left[\varrho_{\epsilon}\right]_{\mathrm{ess}} \frac{\left[s\left(\varrho_{\epsilon}, \vartheta_{\epsilon}\right)\right]_{\mathrm{ess}}-s(\bar{\varrho}, \bar{\vartheta})}{\epsilon} \mathbf{u}_{\epsilon} \rightarrow \bar{\varrho}\left(\frac{\partial s(\bar{\varrho}, \bar{\vartheta})}{\partial \varrho} \varrho^{(1)}+\frac{\partial s(\bar{\varrho}, \bar{\vartheta})}{\partial \vartheta} \vartheta^{(1)}\right) \mathbf{U}
$$

weakly in $L^{2}\left(0, T ; L^{\frac{3}{2}}\left(\Omega ; \mathbb{R}^{3}\right)\right)$.

To complete the proof, we have to check the convergence of

$$
\frac{1}{\epsilon^{2}} \sum_{k=1}^{N} \varrho_{\epsilon} \omega_{k}\left(\vartheta_{\epsilon}, Y_{1}^{\epsilon}, \ldots, Y_{N}^{\epsilon}\right) .
$$


The estimate (5.19) together with (4.4) and (5.16) implies that

$$
0 \leq Y_{k}(t, x) \leq 1, k=1, \ldots, N, \sum_{k=1}^{N} Y_{k}(t, x)=1 \text {, for a.e. }(t, x) \in(0, T) \Omega \text {. }
$$

We next need to control the quantities (5.43). Observe that in accordance with (2.19), (2.20), and the definition of $w_{k}, k=1,2, \ldots, N$ in (1.12),

$$
\begin{aligned}
& \int_{0}^{T} \int_{\Omega}\left|\frac{1}{\epsilon^{2}} \varrho_{\epsilon} \omega_{k}\left(\vartheta_{\epsilon}, Y_{1}^{\epsilon}, \ldots, Y_{N}^{\epsilon}\right)\right| \mathrm{d} x \mathrm{~d} t \leq \int_{0}^{T} \int_{\Omega} \frac{C_{k} G\left(\vartheta_{\epsilon}\right)}{\epsilon} \varrho_{\epsilon}\left|\vartheta_{\epsilon}-\bar{\vartheta}\right| \mathrm{d} x \mathrm{~d} t \\
& \leq \int_{0}^{T} \int_{\Omega} \epsilon C_{k}\left|\frac{\varrho_{\epsilon}-\bar{\varrho}}{\epsilon}\right|\left|\frac{\vartheta_{\epsilon}-\bar{\vartheta}}{\epsilon}\right| \mathrm{d} x \mathrm{~d} t+\int_{0}^{T} \int_{\Omega} \bar{\varrho} C_{k} G\left(\vartheta_{\epsilon}\right)\left|\frac{\vartheta_{\epsilon}-\bar{\vartheta}}{\epsilon}\right| \mathrm{d} x \mathrm{~d} t
\end{aligned}
$$

and the estimates (5.26), (5.28), and (5.29) imply that (5.45) converges to 0 in $L^{p}\left(0, T ; L^{q}(\Omega)\right)$ for certain $p>1, q>1$. Indeed, the last term in (5.45) can be written as

$$
\begin{gathered}
\int_{0}^{T} \int_{\Omega} \bar{\varrho} C_{k} G\left(\vartheta_{\epsilon}\right)\left|\frac{\vartheta_{\epsilon}-\bar{\vartheta}}{\epsilon}\right| \mathrm{d} x \mathrm{~d} t \leq \iint_{\left\{\vartheta_{\epsilon}-\bar{\vartheta} \geq \epsilon K\right\}} \bar{\varrho} C_{k}\left|\frac{\vartheta_{\epsilon}-\bar{\vartheta}}{\epsilon}\right| \mathrm{d} x \mathrm{~d} t \\
+\iint_{\left\{\vartheta_{\epsilon}-\bar{\vartheta}<\epsilon K\right\}} \bar{\varrho} K C_{k} G(\bar{\vartheta}+\epsilon K) \mathrm{d} x \mathrm{~d} t
\end{gathered}
$$

where the first term of the right-hand side converges to 0 as $K \rightarrow \infty$ due to (5.28), (5.29) and the second term of the right-hand side converges to 0 as $\epsilon \rightarrow 0$.

Following the equation (3.10), we can see that

$$
\left\{\varrho_{\epsilon} \frac{Y_{k}^{\epsilon}}{\epsilon}\right\}_{\epsilon>0} \text { is precompact in } C_{\text {weak }}\left([0, T] ; L^{p}(\Omega)\right),
$$

where $p>1$. In accordance with (5.19) and (5.47), we get

$$
\int_{0}^{T} \int_{\Omega} \varrho_{\epsilon}\left(\frac{Y_{k}^{\epsilon}}{\epsilon}\right)^{2} \mathrm{~d} x \mathrm{~d} t \rightarrow \int_{0}^{T} \int_{\Omega} \varrho Y_{k}^{2} \mathrm{~d} x \mathrm{~d} t, k=1,2, \ldots, N
$$

and thus

$$
\frac{Y_{k}^{\epsilon}}{\epsilon} \rightarrow Y_{k} \text { strongly in } L^{2}((0, T) \times \Omega), k=1,2, \ldots, N,
$$

which implies from the estimate (5.22) and (5.45) that, for all $k=1,2, \ldots, N$,

$$
\int_{0}^{T} \int_{\Omega}\left(\bar{\varrho} Y_{k} \partial_{t} \varphi+Y_{k} \mathbf{U} \cdot \nabla_{x} \varphi-D_{k}(\bar{\vartheta}) \nabla_{x} Y_{k} \cdot \nabla_{x} \varphi\right) \mathrm{d} x \mathrm{~d} t=-\int_{\Omega} \bar{\varrho} Y_{k, 0} \varphi(0, \cdot) \mathrm{d} x
$$

for any test function $\varphi \in \mathcal{D}((0, T) \times \bar{\Omega})$, and hence we show (3.17). 
In conclusion, let us take the limit $\epsilon \rightarrow 0$ in the entropy balance equation (5.31) and then we obtain

$$
\begin{aligned}
& \int_{0}^{T} \int_{\Omega} \varrho\left(\frac{\partial s(\bar{\varrho}, \bar{\vartheta})}{\partial \varrho} \varrho^{(1)}+\frac{\partial s(\bar{\varrho}, \bar{\vartheta})}{\partial \vartheta} \vartheta^{(1)}\right)\left(\partial_{t} \varphi+\mathbf{U} \cdot \nabla_{x} \varphi\right) \mathrm{d} x \mathrm{~d} t \\
& -\int_{0}^{T} \int_{\Omega} \frac{\kappa(\bar{\varrho}, \bar{\vartheta})}{\bar{\vartheta}} \nabla_{x} \vartheta^{(1)} \cdot \nabla_{x} \varphi \mathrm{d} x \mathrm{~d} t+\sum_{k=1}^{N} s_{k} \int_{0}^{T} \int_{\Omega} D_{k}(\bar{\vartheta}) \nabla_{x} Y_{k} \cdot \nabla_{x} \varphi \mathrm{d} x \mathrm{~d} t \\
& =-\int_{\Omega} \bar{\varrho}\left(\frac{\partial s(\bar{\varrho}, \bar{\vartheta})}{\partial \varrho} \varrho_{0}^{(1)}+\frac{\partial s(\bar{\varrho}, \bar{\vartheta})}{\partial \vartheta} \vartheta_{0}^{(1)}\right) \varphi(0, \cdot) \mathrm{d} x .
\end{aligned}
$$

We will postpone showing the limit problem of the entropy balance equation in the next section.

5.4. Convergence of the moment equation. To begin with, using two estimates (5.23) and (5.27), we obtain

$$
\varrho_{\epsilon} \mathbf{U}_{\epsilon} \rightarrow \bar{\varrho} \mathbf{U} \text { weakly in } L^{2}\left(0, T ; L^{\frac{30}{23}}\left(\Omega ; \mathbb{R}^{3}\right)\right),
$$

where we have used the Sobolev imbedding theorem $W^{1,2}(\Omega) \hookrightarrow L^{6}(\Omega)$ and it follows from (5.18) that

$$
\varrho_{\epsilon} \mathbf{u}_{\epsilon} \rightarrow \bar{\varrho} \mathbf{U} \text { weakly- } * \text { in } L^{\infty}\left(0, T ; L^{\frac{5}{4}}\left(\Omega ; \mathbb{R}^{3}\right)\right)
$$

Hence

$$
\varrho_{\epsilon} \mathbf{u}_{\epsilon} \otimes \mathbf{u}_{\epsilon} \rightarrow \overline{\varrho \mathbf{U} \otimes \mathbf{U}} \text { weakly in } L^{\infty}\left(0, T ; L^{\frac{30}{23}}\left(\Omega ; \mathbb{R}^{3}\right)\right) .
$$

Actually, due to the oscillations of the gradient component of the velocity field we can not assert at this point that $\overline{\varrho \mathbf{U} \otimes \mathbf{U}}=\varrho \mathbf{U} \otimes \mathbf{U}$ and thus postpone this part to handle the oscillations of the gradient component in the next section.

We now investigate the estimate of temperature. Following (5.17) and (5.29), we get

$$
\left\{\vartheta_{\epsilon}\right\}_{\epsilon>0} \text { is bounded in } L^{\infty}\left(0, T ; L^{4}(\Omega)\right) \cap L^{2}\left(0, T ; L^{6}(\Omega)\right),
$$

which implies that

$$
\mathbb{S}_{\epsilon} \rightarrow \mu(\bar{\vartheta})\left(\nabla_{x} \mathbf{U}+\nabla_{x}^{T} \mathbf{U}\right) \text { weakly in } L^{p}((0, T) \times \Omega)
$$

for a certain $p>1$ thanks to the estimate (5.23) together with the hypothesis of $\mu(2.12)$.

We are now able to identify the limit problem of the moment equation (3.6). Let us take the limit $\epsilon \rightarrow 0$ in the moment equation (3.6) and we get

$$
\begin{aligned}
& \int_{0}^{T} \int_{\Omega}\left(\bar{\varrho} \mathbf{U} \cdot \partial_{t} \varphi+\overline{\varrho \mathbf{U} \otimes \mathbf{U}}: \nabla_{x} \varphi\right) \mathrm{d} x \mathrm{~d} t \\
& =\int_{0}^{T} \int_{\Omega}\left(\mu(\bar{\vartheta})\left(\nabla_{x} \mathbf{U}+\nabla_{x}^{T} \mathbf{U}\right): \nabla_{x} \varphi\right) \mathrm{d} x \mathrm{~d} t-\int_{\Omega}(\varrho \mathbf{U})_{0} \cdot \varphi \mathrm{d} x
\end{aligned}
$$

for any test function

$$
\varphi \in C_{c}^{\infty}\left([0, T] \times \Omega ; \mathbb{R}^{3}\right), \operatorname{div}_{x} \varphi=0,
$$

where we have assumed

$$
\mathbf{u}_{0, \epsilon} \rightarrow \mathbf{U}_{0} \text { weakly- } * \text { in } L^{\infty}\left(\Omega ; \mathbb{R}^{3}\right) .
$$


If we assume $\overline{\varrho \mathbf{U} \otimes \mathbf{U}}=\varrho \overline{\mathbf{U}} \otimes \mathbf{U}$, the initial condition follows as

$$
\mathbf{U}(0, \cdot)=\mathbf{H}\left[\mathbf{U}_{0}\right] .
$$

We next need to handle the pressure and begin with writing

$$
p\left(\varrho_{\epsilon}, \vartheta_{\epsilon}\right)=\left[p\left(\varrho_{\epsilon}, \vartheta_{\epsilon}\right)\right]_{\mathrm{ess}}+\left[p\left(\varrho_{\epsilon}, \vartheta_{\epsilon}\right)\right]_{\mathrm{res}} .
$$

By virtue of (2.5), (2.6), we get

$$
0 \leq \frac{\left[p\left(\varrho_{\epsilon}, \vartheta_{\epsilon}\right)\right]_{\mathrm{res}}}{\epsilon} \leq C\left(\left[\frac{1}{\epsilon}\right]_{\mathrm{res}}+\left[\frac{\varrho_{\epsilon}^{\frac{5}{3}}}{\epsilon}\right]_{\mathrm{res}}+\left[\frac{\vartheta_{\epsilon}^{4}}{\epsilon}\right]_{\mathrm{res}}\right),
$$

which implies from (5.17), (5.19) that

$$
\underset{t \in(0, T)}{\operatorname{ess} \sup }\left\|\frac{\left[p\left(\varrho_{\epsilon}, \vartheta_{\epsilon}\right)\right]_{\mathrm{res}}}{\epsilon}\right\|_{L^{1}(\Omega)} \leq \epsilon C .
$$

From Proposition 5.3 and (5.59), multiplying the momentum equation (3.6) by $\epsilon$ provides

$$
\int_{0}^{T} \int_{\Omega}\left(\frac{\partial p(\bar{\varrho}, \bar{\vartheta})}{\partial \varrho} \varrho^{(1)}+\frac{\partial p(\bar{\varrho}, \bar{\vartheta})}{\partial \vartheta} \vartheta^{(1)}\right) \operatorname{div}_{x} \varphi \mathrm{d} x \mathrm{~d} t=0
$$

for all $\varphi \in C_{c}^{\infty}\left((0, T) \times \Omega ; \mathbb{R}^{3}\right)$. Thus, the relation (5.60) yields

$$
\varrho^{(1)}=-\frac{\partial_{\vartheta} p}{\partial_{\varrho} p}(\bar{\varrho}, \bar{\vartheta}) \vartheta^{(1)}
$$

where we have here used

$$
\int_{\Omega} \varrho^{(1)} \mathrm{d} x=\int_{\Omega} \vartheta^{(1)} \mathrm{d} x=0
$$

Using (5.61) and Gibbs' equation, we obtain

$$
\begin{aligned}
& \int_{0}^{T} \int_{\Omega} \bar{\varrho} c_{p}(\bar{\varrho}, \bar{\vartheta}) \Theta\left(\partial_{t} \varphi+\mathbf{U} \cdot \nabla_{x} \varphi\right) \mathrm{d} x \mathrm{~d} t \\
& -\int_{0}^{T} \int_{\Omega} \frac{\kappa(\bar{\varrho}, \bar{\vartheta})}{\bar{\vartheta}} \nabla_{x} \Theta \cdot \nabla_{x} \varphi \mathrm{d} x \mathrm{~d} t+\sum_{k=1}^{N} s_{k} \int_{0}^{T} \int_{\Omega} D_{k}(\bar{\vartheta}) \nabla_{x} Y_{k} \cdot \nabla_{x} \varphi \mathrm{d} x \mathrm{~d} t \\
& =-\int_{\Omega} \bar{\varrho} c_{p}(\bar{\varrho}, \bar{\vartheta}) \Theta_{0} \varphi(0, \cdot) \mathrm{d} x,
\end{aligned}
$$

where we have here set $\vartheta^{(1)}=\Theta, c_{p}(\bar{\varrho}, \bar{\vartheta}) \Theta_{0}=\vartheta_{0}^{(1)}\left(\frac{\partial s(\bar{\varrho}, \bar{\vartheta})}{\partial \varrho} \varrho_{0}^{(1)}+\frac{\partial s(\bar{\varrho}, \bar{\vartheta})}{\partial \vartheta} \vartheta_{0}^{(1)}\right)$.

5.5. Convergence of the convective term. In general we do not expect

$$
\overline{\varrho \mathbf{U} \otimes \mathbf{U}}=\bar{\varrho} \mathbf{U} \otimes \mathbf{U}
$$

as we mentioned in the previous section, but we may show that (5.63) holds in the weak sense; namely,

$$
\int_{0}^{T} \int_{\Omega} \overline{\varrho \mathbf{U} \otimes \mathbf{U}}: \nabla_{x} \varphi \mathrm{d} x \mathrm{~d} t=\int_{0}^{T} \int_{\Omega}[\bar{\varrho} \mathbf{U} \otimes \mathbf{U}]: \nabla_{x} \varphi \mathrm{d} x \mathrm{~d} t
$$

for any

$$
\varphi \in C_{c}^{\infty}\left((0, T) \times \Omega ; \mathbb{R}^{3}\right), \operatorname{div}_{x} \varphi=0 .
$$


Before we prove (5.64), we will introduce the Helmholtz decomposition, and the following material may be found in most of the textbooks of fluid mechanics.

THEOREM 5.4. A vector function $\mathbf{v}: \Omega \rightarrow \mathbb{R}^{3}$ is written as

$$
\mathbf{v}=\mathbf{H}[\mathbf{v}]+\mathbf{H}^{\perp}[\mathbf{v}]
$$

where

$$
\mathbf{H}^{\perp}[\mathbf{v}]=\nabla_{x} \Phi, \Delta \Phi=\operatorname{div}_{x} \mathbf{v} \text { in } \Omega, \int_{\Omega} \Phi \mathrm{d} x=0 .
$$

Notice that the Helmholtz projections

$$
\mathbf{v} \rightarrow \mathbf{H}[\mathbf{v}] \text { and } \mathbf{v} \rightarrow \mathbf{H}^{\perp}[\mathbf{v}]
$$

map continuously the spaces $L^{p}\left(\Omega ; \mathbb{R}^{3}\right)$ and $W^{1, p}\left(\Omega ; \mathbb{R}^{3}\right)$ into themselves for any $1<p$. We now write

$$
\varrho_{\epsilon} \mathbf{u}_{\epsilon} \otimes \mathbf{u}_{\epsilon}=\mathbf{H}\left[\varrho_{\epsilon} \mathbf{u}_{\epsilon}\right] \otimes \mathbf{u}_{\epsilon}+\mathbf{H}^{\perp}\left[\varrho_{\epsilon} \mathbf{u}_{\epsilon}\right] \otimes \mathbf{H}\left[\mathbf{u}_{\epsilon}\right]+\mathbf{H}^{\perp}\left[\varrho_{\epsilon} \mathbf{u}_{\epsilon}\right] \otimes \mathbf{H}^{\perp}\left[\mathbf{u}_{\epsilon}\right] .
$$

In accordance with the uniform estimates (5.17) and (5.18), we obtain

$$
\mathbf{H}\left[\varrho_{\epsilon} \mathbf{u}_{\epsilon}\right] \rightarrow \mathbf{H}[\varrho \mathbf{U}]=\bar{\varrho} \mathbf{U} \text { in } C_{\text {weak }}\left([0, T] ; L^{\frac{5}{4}}\left(\Omega ; \mathbb{R}^{3}\right)\right) .
$$

In addition, we get

$$
\bar{\varrho} \mathbf{H}\left[\mathbf{u}_{\epsilon}\right] \cdot \mathbf{u}_{\epsilon}=\left(\epsilon \mathbf{H}\left[\frac{\bar{\varrho}-\varrho_{\epsilon}}{\epsilon} \mathbf{u}_{\epsilon}\right]+\mathbf{H}\left[\varrho_{\epsilon} \mathbf{u}_{\epsilon}\right]\right) \cdot \mathbf{u}_{\epsilon} \rightarrow \bar{\varrho}|\mathbf{U}|^{2} \text { weakly in } L^{1}(\Omega)
$$

due to the uniform estimates (5.26) and (5.67). In particular,

$$
\int_{0}^{T} \int_{\Omega}\left|\mathbf{H}\left[\mathbf{u}_{\epsilon}\right]\right|^{2} \mathrm{~d} x \mathrm{~d} t=\int_{0}^{T} \int_{\Omega} \mathbf{H}\left[\mathbf{u}_{\epsilon}\right] \cdot \mathbf{u}_{\epsilon} \mathrm{d} x \mathrm{~d} t \rightarrow \int_{0}^{T} \int_{\Omega}|\mathbf{U}|^{2} \mathrm{~d} x \mathrm{~d} t,
$$

which implies that

$$
\mathbf{H}\left[\mathbf{u}_{\epsilon}\right] \rightarrow \mathbf{U} \text { in } L^{2}\left(0, T ; L^{2}(\Omega)\right) .
$$

Let us first investigate the estimate of $\mathbf{H}\left[\varrho_{\epsilon} \mathbf{u}_{\epsilon}\right] \otimes \mathbf{u}_{\epsilon}$ in (5.66). By virtue of (15.23), (15.67) together with the Sobolev imbedding theorem $W^{1,2}(\Omega) \hookrightarrow L^{5}(\Omega)$, we deduce that

$$
\mathbf{H}\left[\varrho_{\epsilon} \mathbf{u}_{\epsilon}\right] \otimes \mathbf{u}_{\epsilon} \rightarrow \bar{\varrho} \mathbf{U} \otimes \mathbf{U} \text { weakly in } L^{2}\left(0, T ; L^{\frac{30}{29}}(\Omega)\right) .
$$

Moreover, following (5.51) and (5.67), we infer that

$$
\mathbf{H}^{\perp}\left[\varrho_{\epsilon} \mathbf{u}_{\epsilon}\right] \otimes \mathbf{H}\left[\mathbf{u}_{\epsilon}\right] \rightarrow 0 \text { weakly in } L^{2}\left(0, T ; L^{\frac{30}{29}}(\Omega)\right) .
$$

In the previous discussion, it is sufficient to show that

$$
\int_{0}^{T} \int_{\Omega} \mathbf{H}^{\perp}\left[\varrho_{\epsilon} \mathbf{u}_{\epsilon}\right] \otimes \mathbf{H}^{\perp}\left[\mathbf{u}_{\epsilon}\right]: \nabla_{x} \varphi \mathrm{d} x \mathrm{~d} t \rightarrow 0,
$$

for any $\varphi \in C_{c}^{\infty}\left((0, T) \times \Omega ; \mathbb{R}^{3}\right), \operatorname{div}_{x} \varphi=0$ in order to prove (5.63). 
5.6. The acoustic waves. The time evolution of fast acoustic waves for compressible models in the isentropic case was investigated in [2]. In our setting, we have to handle entropic waves as well due to the presence of thermal effects in our system in order to handle the oscillation of $\mathbf{H}^{\perp}\left[\varrho_{\epsilon} \mathbf{u}_{\epsilon}\right]$. To begin with, we write the pressure in the Taylor expansion,

$$
\begin{aligned}
p(\varrho, \vartheta)-p(\bar{\varrho}, \bar{\vartheta})= & \frac{\epsilon}{4}\left(\vartheta^{4}-\bar{\vartheta}^{4}\right)+\frac{\partial p_{F}(\bar{\varrho}, \bar{\vartheta})}{\partial \varrho}(\varrho-\bar{\varrho})+\frac{\partial p_{F}(\bar{\varrho}, \bar{\vartheta})}{\partial \vartheta}(\vartheta-\bar{\vartheta}) \\
& +\left\langle D^{2} p_{F}(a, b)(\varrho-\bar{\varrho}, \vartheta-\bar{\vartheta}),(\varrho-\bar{\varrho}, \vartheta-\bar{\vartheta})\right\rangle
\end{aligned}
$$

and

$$
\begin{aligned}
\varrho(s(\varrho, \vartheta)-s(\bar{\varrho}, \bar{\vartheta}))= & \bar{\varrho} \frac{\partial s(\bar{\varrho}, \bar{\vartheta})}{\partial \varrho}(\varrho-\bar{\varrho})+\bar{\varrho} \frac{\partial s(\bar{\varrho}, \bar{\vartheta})}{\partial \vartheta}(\vartheta-\bar{\vartheta}) \\
& +\left\langle D^{2}(\varrho(s(\varrho, \vartheta)-s(\bar{\varrho}, \bar{\vartheta}))(a, b)(\varrho-\bar{\varrho}, \vartheta-\bar{\vartheta}),(\varrho-\bar{\varrho}, \vartheta-\bar{\vartheta})\rangle,\right.
\end{aligned}
$$

for certain $(a, b)$. In accordance with the Gibb's equation (2.2), one gets

$$
\begin{aligned}
p(\varrho, \vartheta)-p(\bar{\varrho}, \bar{\vartheta})= & \Lambda_{1}(\varrho-\bar{\varrho})+\Lambda_{2}(s(\varrho, \vartheta)-s(\bar{\varrho}, \bar{\vartheta})) \\
& +\left\langle\Sigma\left(a^{\prime}, b^{\prime}\right)(\varrho-\bar{\varrho}, \vartheta-\bar{\vartheta}),(\varrho-\bar{\varrho}, \vartheta-\bar{\vartheta})\right\rangle
\end{aligned}
$$

for a suitable $\Sigma$ where

$$
\Lambda_{1}=\frac{\partial p(\bar{\varrho}, \bar{\vartheta})}{\partial \varrho}+\frac{1}{\bar{\varrho}^{2}}\left(\frac{\partial p(\bar{\varrho}, \bar{\vartheta})}{\partial \vartheta}\right)^{2}\left(\frac{\partial s(\bar{\varrho}, \bar{\vartheta})}{\partial \vartheta}\right)^{-1}, \Lambda_{2}=\frac{1}{\bar{\varrho}}\left(\frac{\partial p(\bar{\varrho}, \bar{\vartheta})}{\partial \vartheta}\right)\left(\frac{\partial s(\bar{\varrho}, \bar{\vartheta})}{\partial \vartheta}\right)^{-1}
$$

We rewrite the dissipative term in (3.5), which will be used in the sequel:

$$
\begin{aligned}
\epsilon \int_{0}^{T} \int_{\Omega} \mathbb{S}_{\epsilon}: \nabla_{x} \varphi \mathrm{d} x \mathrm{~d} t \\
=\epsilon \int_{0}^{T} \int_{\Omega} \mu\left(\vartheta_{\epsilon}\right)\left(\nabla_{x} \mathbf{u}_{\epsilon}+\nabla_{x}^{t} \mathbf{u}_{\epsilon}-\frac{2}{3} \operatorname{div}_{x} \mathbf{u}_{\epsilon} \mathbb{I}\right): \nabla_{x} \varphi \mathrm{d} x \mathrm{~d} t \\
=\epsilon^{2} \int_{0}^{T} \int_{\Omega} \frac{\mu\left(\vartheta_{\epsilon}\right)-\mu(\bar{\vartheta})}{\epsilon}\left(\nabla_{x} \mathbf{u}_{\epsilon}+\nabla_{x}^{t} \mathbf{u}_{\epsilon}-\frac{2}{3} \operatorname{div}_{x} \mathbf{u}_{\epsilon} \mathbb{I}\right): \nabla_{x} \varphi \mathrm{d} x \mathrm{~d} t \\
\quad-2 \epsilon \mu(\bar{\vartheta}) \int_{0}^{T} \int_{\Omega} \mathbf{u}_{\epsilon} \cdot\left(\nabla_{x} \varphi+\nabla_{x}^{t} \varphi-\frac{2}{3} \operatorname{div}_{x} \varphi \mathbb{I}\right) \mathrm{d} x \mathrm{~d} t .
\end{aligned}
$$

We now use the momentum equation (3.5) and the entropy balance (5.31) together with (5.75) to obtain

$$
\begin{aligned}
& \int_{0}^{T} \int_{\Omega}\left(\epsilon r_{\epsilon} \partial_{t} \varphi+\mathbf{V}_{\epsilon} \cdot \nabla_{x} \varphi\right) \mathrm{d} x \mathrm{~d} t \\
& \quad=-\int_{\Omega} \epsilon r_{\epsilon, 0} \varphi(0, \cdot) \mathrm{d} x+\frac{A}{\omega}\left(\int_{0}^{T} \int_{\Omega} \epsilon \Pi_{\epsilon}^{1} \cdot \nabla_{x} \varphi \mathrm{d} x \mathrm{~d} t-\left\langle\sigma_{\epsilon}, \varphi\right\rangle\right)
\end{aligned}
$$


for any $\varphi \in \mathcal{D}([0, T) \times \bar{\Omega})$ and

$$
\begin{aligned}
\int_{0}^{T} \int_{\Omega} \epsilon \mathbf{V}_{\epsilon} & \cdot \partial_{t} \varphi+\omega r_{\epsilon} \operatorname{div}_{x} \varphi+\epsilon D \operatorname{div}_{x}\left(\frac{\nabla_{x} \varphi+\nabla_{x}^{T} \varphi}{2}-\frac{1}{3} \operatorname{div}_{x} \varphi \mathbb{I}\right) \cdot \mathbf{V}_{\epsilon} \mathrm{d} x \mathrm{~d} t \\
= & -\int_{0}^{T} \int_{\Omega} \mathbf{V}_{\epsilon, 0} \cdot \varphi(0, \cdot) \mathrm{d} x+\int_{0}^{T} \int_{\Omega} \Pi_{\epsilon}^{2}: \nabla_{x} \varphi \mathrm{d} x \mathrm{~d} t \\
& +\int_{0}^{T} \int_{\Omega} \Pi_{\epsilon}^{3} \cdot \operatorname{div}_{x}\left(\frac{\nabla_{x} \varphi+\nabla_{x}^{T} \varphi}{2}-\frac{1}{3} \operatorname{div}_{x} \varphi \mathbb{I}\right) \mathrm{d} x \mathrm{~d} t \\
& +\int_{0}^{T} \int_{\Omega} \Pi_{\epsilon}^{4} \operatorname{div}_{x} \varphi \mathrm{d} x \mathrm{~d} t
\end{aligned}
$$

for any test function $\varphi \in \mathcal{D}\left([0, T) \times \Omega ; \mathbb{R}^{3}\right)$, where

$$
\left\{\begin{array}{l}
\omega=\partial_{\varrho} p(\bar{\varrho}, \bar{\vartheta})+\frac{\left|\partial_{\vartheta} p(\bar{\varrho}, \bar{\vartheta})\right|^{2}}{\varrho^{2} \partial_{\vartheta} s(\bar{\varrho}, \bar{\vartheta})}, A=\frac{\partial_{\vartheta} p(\bar{\varrho}, \bar{\vartheta})}{\bar{\varrho} \partial_{\vartheta} s(\bar{\varrho}, \bar{\vartheta})}, D=\frac{2 \mu(\bar{\vartheta})}{\bar{\varrho}}, \\
\mathbf{V}_{\epsilon}=\varrho_{\epsilon} \mathbf{u}_{\epsilon}, \mathbf{V}_{\epsilon, 0}=\varrho_{\epsilon, 0} \mathbf{u}_{\epsilon, 0}, \\
r_{\epsilon}=\frac{1}{\omega}\left(\omega \frac{\varrho_{\epsilon}-\bar{\varrho}}{\epsilon}+A \varrho_{\epsilon} \frac{s\left(\varrho_{\epsilon}, \vartheta_{\epsilon}\right)-s(\bar{\varrho}, \bar{\vartheta})}{\epsilon}\right), \\
r_{\epsilon, 0}=\frac{1}{\omega}\left(\omega \frac{\varrho_{\epsilon, 0}-\bar{\varrho}}{\epsilon}+A \varrho_{\epsilon, 0} \frac{s\left(\varrho_{\epsilon, 0}, \vartheta_{\epsilon, 0}\right)-s(\bar{\varrho}, \bar{\vartheta})}{\epsilon}\right), \\
\Pi_{\epsilon}^{1}=\frac{\kappa\left(\varrho_{\epsilon}, \vartheta_{\epsilon}\right)}{\vartheta_{\epsilon}} \nabla_{x}\left(\frac{\vartheta_{\epsilon}}{\epsilon}\right)-\varrho_{\epsilon} \frac{s\left(\varrho_{\epsilon}, \vartheta_{\epsilon}\right)-s(\bar{\varrho}, \bar{\vartheta})}{\epsilon} \mathbf{u}_{\epsilon}-\frac{1}{\epsilon} \sum_{k=1}^{N} s_{k} \mathcal{F}_{k}, \\
\Pi_{\epsilon}^{2}=\epsilon\left(\mu\left(\vartheta_{\epsilon}\right)-\mu(\vartheta)\right)\left(\nabla_{x} \mathbf{u}_{\epsilon}+\nabla_{x}^{T} \mathbf{u}_{\epsilon}-\frac{2}{3} \operatorname{div}_{x} \mathbf{u}_{\epsilon} \mathbb{I}\right)-\epsilon \varrho_{\epsilon} \mathbf{u}_{\epsilon} \otimes \mathbf{u}_{\epsilon}, \\
\Pi_{\epsilon}^{3}=\omega\left\{\frac{\varrho_{\epsilon}-\bar{\varrho}}{\epsilon}\right\}_{\mathrm{res}}+A\left\{\frac{\varrho_{\epsilon}\left(s\left(\varrho_{\epsilon}, \vartheta_{\epsilon}\right)-s(\bar{\varrho}, \bar{\vartheta})\right)}{\epsilon}\right\}_{\mathrm{res}}-\left\{\frac{p\left(\varrho_{\epsilon}, \vartheta_{\epsilon}\right)-p(\bar{\varrho}, \bar{\vartheta})}{\epsilon}\right\}_{\mathrm{res}} \\
+\epsilon\left\langle\mathbb{A}_{\epsilon}\left\{\frac{\varrho_{\epsilon}-\bar{\varrho}}{\epsilon}, \frac{\vartheta_{\epsilon}-\bar{\vartheta}}{\epsilon}\right\}_{\mathrm{ess}},\left\{\frac{\varrho_{\epsilon}-\bar{\varrho}}{\epsilon}, \frac{\vartheta_{\epsilon}-\bar{\vartheta}}{\epsilon}\right\}_{\mathrm{ess}}\right\rangle,
\end{array}\right.
$$

where $\mathbb{A}_{\epsilon} \in L^{\infty}\left(0, T ; L^{\infty}\left(\Omega ; \mathbb{R}^{2}\right)\right)$.

5.7. Analysis of eigenvalues of the acoustic operator. In this section we study the spectrum of the following differential operator:

$$
\left[\begin{array}{c}
v \\
\mathbf{w}
\end{array}\right] \mapsto \mathcal{A}\left[\begin{array}{c}
v \\
\mathbf{w}
\end{array}\right]+\epsilon \mathcal{B}\left[\begin{array}{c}
v \\
\mathbf{w}
\end{array}\right]
$$

where

$$
\mathcal{A}\left[\begin{array}{c}
v \\
\mathbf{w}
\end{array}\right]=\left[\begin{array}{c}
\omega \operatorname{div}_{x} \mathbf{w} \\
\nabla_{x} v
\end{array}\right], \mathcal{B}\left[\begin{array}{c}
v \\
\mathbf{w}
\end{array}\right]=\left[\begin{array}{c}
0 \\
D \operatorname{div}_{x}\left[\nabla_{x} \mathbf{w}\right]
\end{array}\right] .
$$


By virtue of the no-slip condition, the operator $\mathcal{B}$ is to be supplemented with the boundary conditions

$$
\left.\mathbf{w}\right|_{\partial \Omega}=0
$$

while one takes

$$
\left.\mathbf{w} \cdot \mathbf{n}\right|_{\partial \Omega}=0
$$

for $\mathcal{A}$. We next study the eigenvalue problem

$$
\mathcal{A}\left[\begin{array}{c}
v \\
\mathbf{w}
\end{array}\right]=\lambda\left[\begin{array}{c}
v \\
\mathbf{w}
\end{array}\right] \text { in } \Omega,\left.\mathbf{w}\right|_{\partial \Omega}=0
$$

which can be equivalent to

$$
-\Delta_{x} v=\Lambda v, \Lambda=-\frac{\lambda^{2}}{\omega},\left.\nabla_{x} v\right|_{\partial \Omega}=0
$$

In particular, one can take

$$
\left.\nabla_{x} v \cdot \mathbf{n}\right|_{\partial \Omega}=0
$$

Thus there is a countable set of real eigenvalues $\left\{\Lambda_{n}\right\}_{n=0}^{\infty}$ of the Neumann problem (5.81) and (5.82),

$$
0=\Lambda_{0}<\Lambda_{1}<\Lambda_{2}, \ldots
$$

where the associated family of real eigenfunctions $\left\{v_{n, m}\right\}_{n=0, m=1}^{\infty, m_{n}}$ forms an orthonormal basis of the Hilbert space $L^{2}(\Omega)$. Moreover, we denote the eigenspace by

$$
E_{n}=\operatorname{span}\left\{v_{n, m}\right\}_{m=1}^{m_{n}}, n=0,1, \ldots
$$

Typically, the Neumann problems (5.81) and (5.82) admit a system of eigenvalues

$$
\lambda_{ \pm n}= \pm \mathrm{i} \sqrt{\omega \Lambda_{n}}, n=0,1, \ldots
$$

The associated eigenspace of eigenvalues follows as

$$
\left\{\begin{array}{l}
\operatorname{span}\{1\} \times L_{\sigma}^{2}\left(\Omega ; \mathbb{R}^{3}\right) \text { for } n=0, \\
\operatorname{span}\left\{\left(v_{n, m}, \mathbf{w}_{ \pm n, m}\right)=\frac{1}{\lambda_{ \pm n}} \nabla_{x} v_{n, m}\right\}_{m=1}^{m_{n}} \text { for } n=1,2, \ldots
\end{array}\right.
$$

To simplify our work, for fixed $n>0$, we set

$$
\lambda=\lambda_{n}=\mathrm{i} \sqrt{\omega \Lambda_{n}}, v=v_{n, 1}, \mathbf{w}=\mathbf{w}_{n, 1}=\frac{1}{\lambda_{n}} \nabla_{x} v_{n, 1},
$$

and

$$
E=E_{n}=\operatorname{span}\left\{v_{(1)}, \ldots, v_{(m)}\right\}, v_{(j)}=v_{n, j}, m=m_{n} .
$$

In order to match the incompatibility of the boundary conditions (5.79) and (5.82), we look for approximate eigenfunctions of the following perturbed problem (5.87) and (5.89) in the form

$$
\left\{\begin{array}{l}
v_{\epsilon}=\left(v^{\text {int }, 0}+v^{\mathrm{bl}, 0}\right)+\sqrt{\epsilon}\left(v^{\text {int }, 1}+v^{\mathrm{bl}, 1}\right) \\
\mathbf{w}_{\epsilon}=\left(\mathbf{w}^{\mathrm{int}, 0}+\mathbf{w}^{\mathrm{bl}, 0}\right)+\sqrt{\epsilon}\left(\mathbf{w}^{\mathrm{int}, 1}+v^{\mathrm{bl}, 1}\right),
\end{array}\right.
$$

where we set $v^{\text {int, } 0}=v, \mathbf{w}^{\text {int, } 0}=\mathbf{w}$ and $v_{\epsilon}, \mathbf{w}_{\epsilon}$ are determined as solutions to the following approximate problem:

$$
\left\{\begin{array}{l}
\omega \operatorname{div}_{x} \mathbf{w}_{\epsilon}=\lambda_{\epsilon} v_{\epsilon}+\sqrt{\epsilon} s_{\epsilon}^{1}, \\
\nabla_{x} v_{\epsilon}+\epsilon D \operatorname{div}_{x}\left[\left[\nabla_{x} \mathbf{w}_{\epsilon}\right]\right]=\lambda_{\epsilon} \mathbf{w}_{\epsilon}+\sqrt{\epsilon} s_{\epsilon}^{2},
\end{array}\right.
$$


where

$$
[[\mathbb{M}]]=\frac{\mathbb{M}+\mathbb{M}^{T}}{2}-\frac{1}{3} \operatorname{trace}(\mathbb{M}) \mathbb{I}
$$

and

$$
\lambda_{\epsilon}=\lambda^{0}+\sqrt{\epsilon} \lambda^{1}, \text { with } \lambda^{0}=\lambda
$$

supplemented with the homogeneous Dirichlet boundary condition

$$
\left.\mathbf{w}_{\epsilon}\right|_{\partial \Omega}=0 \text {. }
$$

In the spirit of [6] we have that

$$
\left\{\begin{array}{l}
\operatorname{Re}\left[\lambda^{1}\right]<0, \\
s_{\epsilon}^{1} \rightarrow 0 \text { in } L^{p}(\Omega), s_{\epsilon}^{2} \rightarrow 0 \text { in } L^{p}\left(\Omega ; \mathbb{R}^{3}\right) \text { as } \epsilon \rightarrow 0, p>1,
\end{array}\right.
$$

which plays an important role in the proof of strong convergence of $\mathbf{u}_{\epsilon}$ in the next section.

5.8. Strong convergence of velocity. In view of the previous result, it only remains to prove that

$$
\mathbf{H}^{\perp}\left[\mathbf{u}_{\epsilon}\right] \rightarrow 0 \text { (strongly) in } L^{2}\left(0, T ; L^{2}\left(\Omega ; \mathbb{R}^{3}\right)\right) .
$$

Indeed, it is sufficient to show that

$$
\left[t \mapsto \int_{\Omega} \mathbf{u}_{\epsilon} \cdot \mathbf{w} \mathrm{d} x\right] \rightarrow 0 \text { in } L^{2}(0, T)
$$

for any fixed $\mathbf{w}=\frac{1}{\lambda} \nabla_{x} v$, where $\{v, \mathbf{w}, \lambda\}$ solves the eigenvalue problem (5.81). Moreover, if we show that

$$
\left[t \mapsto \int_{\Omega} \varrho_{\epsilon} \mathbf{u}_{\epsilon} \cdot \mathbf{w d} x\right] \rightarrow 0 \text { in } L^{2}(0, T),
$$

the relation (5.93) holds thanks to the convergence of $\varrho_{\epsilon}$ in (5.27). In addition, taking into consideration the eigenvalue problem (5.81) and (5.82) and the pairs $[v, \mathbf{w}, \lambda],[v,-\mathbf{w},-\lambda]$, it is enough to show that

$$
\left[t \mapsto \int_{\Omega}\left(r_{\epsilon} v_{\epsilon}+\mathbf{V}_{\epsilon} \cdot \mathbf{w}_{\epsilon}\right) \mathrm{d} x\right] \rightarrow 0 \text { in } L^{2}(0, T),
$$

in order to prove (5.93) where, in virtue of (5.90), we have here used

$$
v_{\epsilon} \rightarrow v \text { in } C(\bar{\Omega}), \mathbf{w}_{\epsilon} \rightarrow \mathbf{w} \text { in } L^{p}\left(\Omega ; \mathbb{R}^{3}\right)
$$

for any $1 \leq p<\infty$. We adopt test functions $\psi(t) v_{\epsilon}(x), \psi(t) \mathbf{w}_{\epsilon}(x), \phi \in C_{c}^{\infty}(0, T)$ in the acoustic equations (5.76), (5.77), respectively, and then we write

$$
\int_{0}^{T}\left(\epsilon \zeta_{\epsilon} \partial_{t} \phi+\lambda_{\epsilon} \zeta_{\epsilon} \phi\right) \mathrm{d} t+\sqrt{\epsilon} \int_{0}^{T} \psi \int_{\Omega}\left(r_{\epsilon} s_{\epsilon}^{1}+\mathbf{V}_{\epsilon} \cdot s_{\epsilon}^{2}\right) \mathrm{d} x \mathrm{~d} t=\sum_{k=1}^{6} I_{\epsilon}^{k},
$$

where

$$
\zeta_{\epsilon}(t)=\int_{\Omega}\left(r_{\epsilon}(t, \cdot) v_{\epsilon}+\mathbf{V}_{\epsilon}(t, \cdot) \cdot \mathbf{w}_{\epsilon}\right) \mathrm{d} x
$$


and

$$
\left\{\begin{array}{l}
I_{\epsilon}^{1}=\frac{A}{\omega} \int_{0}^{T} \int_{\Omega} \Pi_{\epsilon}^{1} \cdot \nabla_{x} v_{\epsilon} \psi \mathrm{d} x \mathrm{~d} t \\
I_{\epsilon}^{2}=-\frac{A}{\omega}\left\langle\sigma_{\epsilon}+\sum_{k=1}^{N} \frac{h_{k}}{\epsilon^{2} \vartheta_{\epsilon}} \varrho_{\epsilon} \omega_{k}\left(\vartheta_{\epsilon}, Y_{1}^{\epsilon}, \ldots, Y_{N}^{\epsilon}\right), v_{\epsilon} \psi\right\rangle, \\
I_{\epsilon}^{3}=\frac{A}{\omega} \int_{0}^{T} \int_{\Omega} \sum_{k=1}^{N} \frac{h_{k}}{\epsilon^{2} \vartheta_{\epsilon}} \varrho_{\epsilon} \omega_{k}\left(\vartheta_{\epsilon}, Y_{1}^{\epsilon}, \ldots, Y_{N}^{\epsilon}\right) v_{\epsilon} \psi \mathrm{d} x \mathrm{~d} t \\
I_{\epsilon}^{4}=\int_{0}^{T} \int_{\Omega} \Pi_{\epsilon}^{2}: \nabla_{x} \mathbf{w}_{\epsilon} \psi \mathrm{d} x \mathrm{~d} t \\
I_{\epsilon}^{5}=D \epsilon \int_{0}^{T} \int_{\Omega}\left(\varrho_{\epsilon}-\bar{\varrho}\right) \operatorname{div}{ }_{x}\left[\left[\nabla_{x} \mathbf{w}_{\epsilon}\right]\right] \cdot \mathbf{u}_{\epsilon} \psi \mathrm{d} x \mathrm{~d} t, \\
I_{\epsilon}^{6}=\int_{0}^{T} \int_{\Omega} \Pi_{\epsilon}^{3} \operatorname{div}_{x} \mathbf{w}_{\epsilon} \psi \mathrm{d} x \mathrm{~d} t
\end{array}\right.
$$

with $\Pi_{\epsilon}^{1}, \Pi_{\epsilon}^{2}, \Pi_{\epsilon}^{3}$ defined in (5.78).

To complete the proof, it is sufficient to handle the terms $I_{\epsilon}^{k}, k=1, \ldots, 6$, in order to show (5.94) where we have here used (5.90). Indeed, we get suitable estimates of $I_{\epsilon}^{k}, k=1,4,5,6$, with the same process as Section 6.1 in 8 . It remains to handle $I_{\epsilon}^{2}, I_{\epsilon}^{3}$. In virtue of the estimate (5.18), one gets

$$
I_{\epsilon}^{2}=\epsilon^{2}<\Gamma^{\epsilon}, \psi>_{[\mathcal{M} ; C][0, T]},
$$

where $\left\{\Gamma^{\epsilon}\right\}_{\epsilon>0}$ is bounded in $\mathcal{M}^{+}[0, T]$. For $I_{\epsilon}^{3}$, we can use the estimate (5.45) to obtain

$$
I_{\epsilon}^{3}=\epsilon \int_{0}^{T} \psi(t) \alpha_{\epsilon}(t) \mathrm{d} t
$$

where $\left\{\alpha_{\epsilon}\right\}_{\epsilon>0}$ is bounded in $L^{p}(0, T)$ for a certain $p>1$. We can see the details of the proofs of other uniform bounds in Section 7.5 of Feireisl, Novotný [6].

We now come back to the proof of (5.94). To this end, we regularize equation (5.95) with respect to the time variable using the standard regularizing kernels $\theta \in C_{c}^{\infty}(-1,1)$ satisfying

$$
\theta_{\delta}(t)=\frac{1}{\delta} \theta\left(\frac{t}{\delta}\right), \theta \geq 0, \int_{-1}^{1} \theta(t) \mathrm{d} t=1
$$

and then it follows that

$$
\frac{\mathrm{d}}{\mathrm{d} t} \zeta_{\epsilon, \delta}-\frac{\lambda_{\epsilon}}{\epsilon} \zeta_{\epsilon, \delta}=\sqrt{\epsilon} \pi_{\epsilon, \delta}^{1}+\pi_{\epsilon, \delta}^{2}+\frac{1}{\sqrt{\epsilon}} \pi_{\epsilon, \delta}^{3},
$$


where, in accordance with the all-uniform bounds of $I_{\epsilon}^{k}, k=1, \ldots, 6$, we have

$$
\left\{\begin{array}{l}
\left\|\pi_{\epsilon, \delta}^{1}\right\|_{L^{1}(0, T)} \leq C, \\
\left\|\pi_{\epsilon, \delta}^{2}\right\|_{L^{p}(0, T)} \leq C, p>1, \\
\lim _{\epsilon \rightarrow 0} \sup _{\delta>0}\left\|\pi_{\epsilon, \delta}^{3}\right\|_{L^{\infty}(0, T)}=0 .
\end{array}\right.
$$

The standard variation of constants formula now yields

$$
\begin{aligned}
\left|\zeta_{\epsilon, \delta}(t)\right| \leq & \exp \left(\operatorname{Re}\left[\lambda_{\epsilon} / \epsilon\right](t-\delta)\right) \underset{s \in(0, T)}{\operatorname{ess} \sup }\left|\zeta_{\epsilon, \delta}(s)\right|+\sqrt{\epsilon} \int_{0}^{T}\left|\pi_{\epsilon, \delta}^{1}(s)\right| d s \\
& +\int_{0}^{T} \exp \left(\operatorname{Re}\left[\lambda_{\epsilon} / \epsilon\right](t-s)\right)\left|\pi_{\epsilon, \delta}^{2}(s)\right| d s \\
& +\int_{0}^{T} \frac{1}{\sqrt{\epsilon}} \exp \left(\operatorname{Re}\left[\lambda_{\epsilon} / \epsilon\right](t-s)\right)\left|\pi_{\epsilon, \delta}^{3}(s)\right| d s
\end{aligned}
$$

where we have used

$$
\operatorname{Re}\left[\lambda_{\epsilon} / \epsilon\right] \leq-\frac{C}{\sqrt{\epsilon}}
$$

derived from (5.90) and $\lambda_{\epsilon}=\lambda_{0}+\sqrt{\epsilon} \lambda^{1}$. By virtue of (5.100), we easily see that

$$
\int_{0}^{T} \frac{1}{\sqrt{\epsilon}} \exp \left(\operatorname{Re}\left[\lambda_{\epsilon} / \epsilon\right](t-s)\right) \mid d s<C .
$$

Consequently, letting $\epsilon \rightarrow 0$ and passing to the limit $\delta \rightarrow 0$ together with (5.98) and (5.101), we prove (5.94).

We now check the boundary conditions of the target system given in (3.2). Since $\mathbf{u}_{\epsilon}$ converges to $\mathbf{U}$ in $L^{2}\left(0, T ; L^{2}\left(\Omega ; \mathbb{R}^{3}\right)\right)$, we get $\mathbf{U}=0$ on $\partial \Omega$. In accordance with the estimates (5.22), (5.28), and (5.55), we conclude that

$$
\mathbb{S} \mathbf{n} \times \mathbf{n}=0, \mathcal{F}_{k} \cdot \mathbf{n}=0, k=1,2, \ldots, N, \nabla_{x} \Theta \cdot \mathbf{n}=0 \text { in the sense of trace, }
$$

which verify the boundary conditions given in (3.13).

6. Low Mach number on the unbounded domains. The only difference between the proofs of Theorem 4.1 and Theorem 4.2 is in showing (5.94) for the gradient part $\mathbf{H}^{\perp}\left[\mathbf{V}_{\epsilon}\right]$. In this section we follow the framework of Feireisl [4] based on Kato's result [12, Theorem 6.1

Theorem 6.1 (see Reed and Simon [17]). Let $A$ be a closed densely defined linear operator, and let $H$ be a self-adjoint densely defined operator in a Hilbert space $M$. For $\lambda \in \mathbb{C}-\mathbb{R}$, let $R_{H}[\lambda]=(H-\lambda \mathrm{Id})^{-1}$ denote the resolvent of $H$. Suppose that

$$
\Gamma:=\sup _{\lambda \in \mathbb{C}-\mathbb{R}, v \in \mathcal{D}\left(A^{*}\right),\|v\|=1}\left\|A \circ R_{H}[\lambda] \circ A^{*}[v]\right\|_{X}<\infty .
$$

Then

$$
\sup _{w \in X,\|w\|_{X}=1} \frac{\pi}{2} \int_{-\infty}^{\infty}\|A \exp (-\mathrm{i} t H)[w]\|_{X}^{2} \mathrm{~d} t \leq \Gamma^{2} .
$$


We now introduce a "time lifting" $\Sigma_{\epsilon}$ of the measure $\sigma_{\epsilon}$ by

$$
\left\langle\Sigma_{\epsilon}, \varphi\right\rangle:=\left\langle\sigma_{\epsilon}, I[\varphi]\right\rangle,
$$

where

$$
I[\varphi](t, x):=\int_{0}^{t} \varphi(s, x) d s
$$

for any $\varphi \in L^{1}\left(0, T ; \Omega_{\epsilon}\right)$. It is easy to see that $\Sigma_{\epsilon} \in L_{\text {weak }}^{\infty}\left(0, T ; \mathcal{M}^{+}\left(\bar{\Omega}_{\epsilon}\right)\right)$, where

$$
\left\langle\Sigma_{\epsilon}(\tau), \varphi\right\rangle:=\lim _{\delta \rightarrow 0+}\left\langle\sigma_{\epsilon}, \psi_{\delta} \varphi\right\rangle,
$$

with

$$
\psi_{\delta}(t)= \begin{cases}0 & \text { for } t \in[0, \tau), \\ \frac{1}{\delta}(t-\tau) & \text { for } t \in(\tau, \tau+\delta), \\ 1 & \text { for } t \geq \tau+\delta .\end{cases}
$$

Using the acoustic equation (5.77), (5.78), we rewrite it as

$$
\left\{\begin{array}{l}
\epsilon \partial_{t} X_{\epsilon}+\operatorname{div}_{x} \mathbf{V}_{\epsilon}=\epsilon \operatorname{div}_{x} \mathbf{F}_{\epsilon}^{1}, \\
\epsilon \partial_{t} \mathbf{V}_{\epsilon}+\omega \nabla_{x} X_{\epsilon}=\epsilon\left(\operatorname{div}_{x} \mathbf{F}_{\epsilon}^{2}+\nabla_{x} \mathbf{F}_{\epsilon}^{3}+\frac{A}{\epsilon^{2} \omega} \nabla_{x} \Sigma_{\epsilon}\right)
\end{array}\right.
$$

with the boundary condition $\left.\mathbf{V}_{\epsilon} \cdot \mathbf{n}\right|_{\partial \Omega_{\epsilon}}=0$, where

$$
\left\{\begin{array}{l}
X_{\epsilon}=r_{\epsilon}+\frac{A}{\epsilon^{2} \omega} \Sigma_{\epsilon}, \mathbf{V}_{\epsilon}=\varrho_{\epsilon} \mathbf{u}_{\epsilon}, \\
\mathbf{F}_{\epsilon}^{1}=\Pi_{\epsilon}^{1}, \mathbf{F}_{\epsilon}^{2}=\mathbb{S}_{\epsilon}-\varrho_{\epsilon} \mathbf{u}_{\epsilon} \otimes \mathbf{u}_{\epsilon}, \\
\mathbf{F}_{\epsilon}^{3}=\frac{r_{\epsilon}}{\epsilon}-\left(\frac{p\left(\varrho_{\epsilon}, \vartheta_{\epsilon}\right)-p(\bar{\varrho}, \bar{\vartheta})}{\epsilon^{2}}\right) .
\end{array}\right.
$$

We now regularize $X_{\epsilon}, \mathbf{V}_{\epsilon}, \mathbf{F}_{\epsilon}^{1}, \mathbf{F}_{\epsilon}^{2}, \mathbf{F}_{\epsilon}^{3}$ with respect to the variable $x$ on $\partial \Omega_{\epsilon}$ in order to extend them on $\Omega$ in the spirit of Feireisl [4, and we denote by

$$
X_{\epsilon, \delta}, \mathbf{V}_{\epsilon, \delta}, \mathbf{F}_{\epsilon, \delta}^{1}, \mathbf{F}_{\epsilon, \delta}^{2}, \mathbf{F}_{\epsilon, \delta}^{3}
$$

the regularized quantities. Notice that the uniform estimates (5.19), (5.28), (5.29) with (2.15) imply

$$
\Pi_{\epsilon, \delta}^{1} \rightarrow \Pi_{\epsilon}^{1} \text { in } L^{2}\left(0, T ; L^{1}\left(\Omega_{\epsilon} ; \mathbb{R}^{3}\right)\right),
$$

as $\delta \rightarrow 0$ where we have used the following inequality:

$$
\int_{\Omega}\left|\frac{D_{k} \nabla_{x} Y_{k}}{\epsilon}\right| \mathrm{d} x \leq \int_{\Omega}\left(1+2 \vartheta_{\epsilon}^{\alpha}+\vartheta_{\epsilon}^{2 \alpha} \mathrm{d} x\right)^{1 / 2} \times\left(\int_{\Omega}\left|\frac{\nabla_{x} Y_{k}}{\epsilon}\right|^{2} \mathrm{~d} x\right)^{1 / 2}
$$

for $k=1,2, \ldots, N$.

Consider the following initial-boundary-value problem:

$$
\left\{\begin{array}{l}
\epsilon \partial_{t} X_{\epsilon}+\operatorname{div}_{x} \mathbf{V}_{\epsilon}=\epsilon \operatorname{div}_{x} \mathbf{F}_{\epsilon}^{1} \text { in }(0, T) \times \Omega, \\
\epsilon \partial_{t} \mathbf{V}_{\epsilon}+\omega \nabla_{x} X_{\epsilon}=\epsilon \operatorname{div}_{x} \mathbf{F}_{\epsilon}^{2} \text { in }(0, T) \times \Omega,
\end{array}\right.
$$


with the boundary condition $\left.\mathbf{V}_{\epsilon} \cdot \mathbf{n}\right|_{\partial \Omega}=0$ and the initial conditions $X_{\epsilon}(0, \cdot)=X_{0, \epsilon}$, $\mathbf{V}_{\epsilon}(0, \cdot)=\mathbf{V}_{0, \epsilon}$ in $\Omega$ and observe that

$$
\underset{t \in(0, T)}{\operatorname{ess} \sup _{0}}\left|\int_{\Omega}\left(\mathbf{V}_{\epsilon, \delta}-\mathbf{V}_{\epsilon}\right)(t, \cdot) \cdot \mathbf{w d} x\right| \rightarrow 0 \text { as } \delta \rightarrow 0 .
$$

In view of $\mathbf{H}^{\perp}\left[\mathbf{V}_{\epsilon}\right]=\nabla \Phi_{\epsilon}$, the initial-boundary-value problem (16.6) can be written as

$$
\left\{\begin{array}{l}
\epsilon \partial_{t} X_{\epsilon}+\Delta \Phi_{\epsilon}=\epsilon \operatorname{div}_{x} \mathbf{F}_{\epsilon}^{1} \text { in }(0, T) \times \Omega, \\
\epsilon \partial_{t} \Phi_{\epsilon}+\omega X_{\epsilon}=\epsilon \Delta^{-1}\left[\operatorname{div}_{x} \operatorname{div}_{x} \mathbf{F}_{\epsilon}^{2}\right] \text { in }(0, T) \times \Omega,
\end{array}\right.
$$

with the boundary condition $\left.\nabla_{X} \Phi_{\epsilon} \cdot \mathbf{n}\right|_{\partial \Omega}=0$ and the initial conditions $X_{\epsilon}(0, \cdot)=$ $X_{0, \epsilon}, \Phi_{\epsilon}(0, \cdot)=\Phi_{0, \epsilon}=\Delta^{-1}\left[\operatorname{div}_{x} \mathbf{V}_{0, \epsilon}\right]$ in $\Omega$. From Duhamel's formula used to solve the initial-boundary-value problem (6.7), we get

$$
\begin{aligned}
\Phi_{\epsilon}(t)= & \exp \left(\mathrm{i} \frac{t}{\epsilon} \sqrt{-\Delta}\right)\left[\frac{1}{2} \Phi_{0, \epsilon}+\frac{\mathrm{i}}{2 \sqrt{-\Delta}}\left[X_{0, \epsilon}\right]\right] \\
& +\exp \left(-\mathrm{i} \frac{t}{\epsilon} \sqrt{-\Delta}\right)\left[\frac{1}{2} \Phi_{0, \epsilon}-\frac{\mathrm{i}}{2 \sqrt{-\Delta}}\left[X_{0, \epsilon}\right]\right] \\
& +\int_{0}^{t} \exp \left(\mathrm{i} \frac{t-s}{\epsilon} \sqrt{-\Delta}\right)\left[\frac{1}{2} \frac{1}{\Delta} \operatorname{div}_{x} \operatorname{div}_{x} \mathbf{F}_{\epsilon}^{2}+\frac{\mathrm{i}}{2 \sqrt{-\Delta}}\left[\operatorname{div}_{x} \mathbf{F}_{\epsilon}^{1}\right]\right] d s \\
& +\int_{0}^{t} \exp \left(-\mathrm{i} \frac{t-s}{\epsilon} \sqrt{-\Delta}\right)\left[\frac{1}{2} \frac{1}{\Delta} \operatorname{div}_{x} \operatorname{div}_{x} \mathbf{F}_{\epsilon}^{2}-\frac{\mathrm{i}}{2 \sqrt{-\Delta}}\left[\operatorname{div}_{x} \mathbf{F}_{\epsilon}^{1}\right]\right] d s
\end{aligned}
$$

Following a similar line of argument as in Feireisl [4, the desired result (5.94) is established for the gradient part $\mathbf{H}^{\perp}\left[\mathbf{V}_{\epsilon}\right]$ by applying Theorem 6.1 with $M=L^{2}(\Omega), H=$ $\sqrt{-\Delta}, A=\varphi G(-\Delta), \varphi \in C_{c}^{\infty}(\Omega), G \in C_{c}^{\infty}(0, \infty)$. Indeed,

$$
\begin{aligned}
& \int_{\Omega} \nabla_{x} \Phi_{\epsilon} \cdot \mathbf{w d} x=-\int_{\Omega} \Phi_{\epsilon} \operatorname{div}_{x} \mathbf{w} \mathrm{d} x=-\int_{\Omega} \varphi \Phi_{\epsilon} \operatorname{div}_{x} \mathbf{w} \mathrm{d} x \\
&=-\int_{\Omega} \varphi G(-\Delta)\left[\Phi_{\epsilon}\right] \operatorname{div}_{x} \mathbf{w} \mathrm{d} x+\int_{\Omega} \varphi(G(-\Delta)-\mathrm{Id})\left[\Phi_{\epsilon}\right] \operatorname{div}_{x} \mathbf{w} \mathrm{d} x,
\end{aligned}
$$

which converges to 0 as $\epsilon$ tends to 0 .

\section{REFERENCES}

[1] T.R. Bose. High temperature gas dynamics. Springer-Verlag, Berlin, 2004.

[2] B. Desjardins, E. Grenier, P.-L. Lions, and N. Masmoudi. Incompressible limit for solutions of the isentropic Navier-Stokes equations with Dirichlet boundary conditions. J. Math. Pures Appl., 78 461-471, 1999. MR1697038 (2000j:35220)

[3] S. Eliezer, A. Ghatak, and H. Hora. An introduction to equations of states, theory and applications. Cambridge University Press, Cambridge, 1986.

[4] E. Feireisl. Incompressible limits and propagation of acoustic waves in large domains with boundaries. Comm. Math. Phys., 294 (1) 73-95, 2010. MR2575476 (2011a:35410)

[5] E. Feireisl. Stability of flows of real monoatomic gases. Commun. Partial Differential Equations, 31:325-348, 2006. MR2209757 (2007f:35216)

[6] E. Feireisl and A. Novotný. Singular limits in thermodynamics of viscous fluids. Birkhäuser Verlag, Basel, 2009. MR2499296 (2011b:35001)

[7] E. Feireisl and A. Novotný. The low Mach number limit for the full Navier-Stokes-Fourier system. Arch. Ration. Mech. Anal.,186(1):77-107 2007. MR2338352 (2008m:35277)

[8] E. Feireisl and H. Petzeltová. Low Mach number asymptotics for reacting compressible fluid flows. Discrete Contin. Dyn. Syst., 26(2):455-480, 2010. MR2556494 (2010m:35388)

[9] E. Feireisl, H. Petzeltová, and K. Trivisa. Multicomponent reactive flows: global-in-time existence for large data. Commun. Pure Appl. Anal. 7, no. 5, (2008), 1017-1047. MR2410865(2009k:35232) 
[10] G. Gallavotti. Statistical mechanics: A short treatise. Springer-Verlag, Heidelberg, 1999. MR 1707309 (2001j:82001)

[11] V. Giovangigli. Multicomponent flow modeling. Birkhäuser, Basel, 1999. MR.1713516 (2000h:76168)

[12] T. Kato. Wave operators and similarity for some non-selfadjoint operators. Math. Ann., 162:258279, 1965/1966. MR0190801 (32:8211)

[13] S. Klainerman and A. Majda. Singular limits of quasilinear hyperbolic systems with large parameters and the incompressible limit of compressible fluids. Comm. Pure Appl. Math., 34:481-524, 1981. MR615627 (84d:35089)

[14] R. Klein, N. Botta, T. Schneider, C.D. Munz, S. Roller, A. Meister, L. Hoffmann, and T. Sonar. Asymptotic adaptive methods for multi-scale problems in fluid mechanics. J. Engrg. Math., 39:261343, 2001. MR 1826065 (2002a:76118)

[15] P.-L. Lions and N. Masmoudi. Incompressible limit for a viscous compressible fluid J. Math. Pures Appl. (9), 77:585-627, 1998. MR.1628173 (99d:76084)

[16] J. Oxenius. Kinetic theory of particles and photons. Springer-Verlag, Berlin, 1986. MR835211 $(87 \mathrm{~m}: 82051)$

[17] M. Reed and B. Simon. Methods of Modern Mathematical Physics.IV. Analysis of operators. New York: Academic Press [Harcourt Brace Jovanovich Publishers], 1978. MR0493421 (58:12429c) 